\title{
AN ULTRAVIOLET-TO-RADIO BROADBAND SPECTRAL ATLAS OF NEARBY GALAXIES
}

\author{
D. A. Dale, ${ }^{1}$ A. Gil de Paz, ${ }^{2}$ K. D. Gordon, ${ }^{3}$ H. M. Hanson, ${ }^{1}$ L. Armus, ${ }^{4}$ G. J. Bendo, ${ }^{5}$ L. Bianchi, ${ }^{6}$ M. Block, ${ }^{3}$ \\ S. Boissier, ${ }^{7,8}$ A. Boselli, ${ }^{8}$ B. A. Buckalew, ${ }^{9}$ V. Buat,${ }^{8}$ D. Burgarella, ${ }^{8}$ D. Calzetti, ${ }^{10}$ J. M. Cannon, ${ }^{11}$ \\ C. W. Engelbracht, ${ }^{3}$ G. Helou, ${ }^{9}$ D. J. Hollenbach, ${ }^{12}$ T. H. Jarrett, ${ }^{4}$ R. C. Kennicutt, ${ }^{3,13}$ \\ C. Leitherer, ${ }^{10}$ A. Li, ${ }^{14}$ B. F. Madore, ${ }^{7}$ D. C. Martin, ${ }^{15}$ M. J. Meyer, ${ }^{10}$ \\ E. J. Murphy, ${ }^{16}$ M. W. Regan, ${ }^{10}$ H. Roussel, ${ }^{17}$ J. D. T. Smith, ${ }^{3}$ \\ M. L. Sosey, ${ }^{10}$ D. A. Thilker, ${ }^{6}$ AND F. Walter ${ }^{17}$ \\ Received 2006 September 25; accepted 2006 October 20
}

\begin{abstract}
The ultraviolet-to-radio continuum spectral energy distributions are presented for all 75 galaxies in the Spitzer Infrared Nearby Galaxies Survey (SINGS). A principal component analysis of the sample shows that most of the sample's spectral variations stem from two underlying components, one representative of a galaxy with a low infrared-toultraviolet ratio and one representative of a galaxy with a high infrared-to-ultraviolet ratio. The influence of several parameters on the infrared-to-ultraviolet ratio is studied (e.g., optical morphology, disk inclination, far-infrared color, ultraviolet spectral slope, and star formation history). Consistent with our understanding of normal star-forming galaxies, the SINGS sample of galaxies in comparison to more actively star-forming galaxies exhibits a larger dispersion in the infrared-to-ultraviolet versus ultraviolet spectral slope correlation. Early-type galaxies, exhibiting low star formation rates and high optical surface brightnesses, have the most discrepant infrared-to-ultraviolet correlation. These results suggest that the star formation history may be the dominant regulator of the broadband spectral variations between galaxies. Finally, a new discovery shows that the $24 \mu \mathrm{m}$ morphology can be a useful tool for parameterizing the global dust temperature and ultraviolet extinction in nearby galaxies. The dust emission in dwarf/irregular galaxies is clumpy and warm accompanied by low ultraviolet extinction, while in spiral galaxies there is typically a much larger diffuse component of cooler dust and average ultraviolet extinction. For galaxies with nuclear $24 \mu \mathrm{m}$ emission, the dust temperature and ultraviolet extinction are relatively high compared to disk galaxies.
\end{abstract}

Subject headings: galaxies: photometry — infrared: galaxies — infrared: ISM — ultraviolet: galaxies

Online material: color figures

\section{INTRODUCTION}

Interstellar dust has always presented challenges to astronomers. Extinction makes it difficult to extract intrinsic fluxes. Reddening leads to uncertain colors. An outstanding challenge is to identify dust absorption features (diffuse interstellar bands) that were discovered over 80 years ago. Nonetheless, interstellar dust also provides unique opportunities for understanding galaxy structure and evolution. The formation of molecules, interstellar heating and cooling processes, polarization, and photometric redshift indicators are just a few of the areas of study that benefit from

1 Department of Physics and Astronomy, University of Wyoming, Laramie, WY; ddale@uwyo.edu.

${ }^{2}$ Departamento de Astrofisica, Universidad Complutense, Madrid, Spain.

3 Steward Observatory, University of Arizona, Tucson, AZ.

${ }^{4}$ Spitzer Science Center, California Institute of Technology, Pasadena, CA.

5 Astrophysics Group, Imperial College, Blackett Laboratory, London, UK.

${ }^{6}$ Center for Astrophysical Sciences, Johns Hopkins University, Baltimore, $\mathrm{MD}$.

${ }^{7}$ Carnegie Observatories, Carnegie Institution of Washington, Pasadena, CA.

${ }^{8}$ Laboratoire d'Astrophysique de Marseille, Traverse du Siphon, Marseille, France.

${ }^{9}$ California Institute of Technology, Pasadena, CA.

${ }_{10}$ Space Telescope Science Institute, Baltimore, MD.

11 Astronomy Department, Wesleyan University, Middletown, CT.

12 NASA/Ames Research Center, Moffett Field, CA.

13 Institute of Astronomy, University of Cambridge, Cambridge, UK.

${ }^{14}$ Department of Physics and Astronomy, University of Missouri, Columbia, MO.

${ }^{15}$ Astronomy Option, California Institute of Technology, Pasadena, CA

${ }^{16}$ Department of Astronomy, Yale University, New Haven, CT.

17 Max-Planck-Institut für Astronomie, Heidelberg, Germany. the presence and knowledge of interstellar grains (for a review see Draine 2003).

Although dust primarily releases energy over infrared and submillimeter wavelengths, much of the radiation intercepted by interstellar grains originates in the ultraviolet from the atmospheres of OB stars. Thus, the combination of infrared and ultraviolet data should provide a powerful diagnostic of star formation and selective extinction. One important application is determining ultraviolet-based star formation rates corrected for dust extinction. High-redshift surveys carried out in the rest-frame ultraviolet and optical, for example, are particularly vulnerable to the presence of interstellar dust (e.g., Adelberger \& Steidel 2000). Fortunately, studies coupling infrared and ultraviolet data have shown that the slope of the ultraviolet continuum is one such useful probe of the extinction in starburst galaxies (e.g., Calzetti et al. 1994; Meurer et al. 1999). Subsequent work in this area has explored how the infrared-to-ultraviolet ratio and its scatter depend on bolometric and monochromatic luminosity, ultraviolet spectral slope, metallicity, diameter, star formation rate, etc. (e.g., Gordon et al. 2000, 2004; Buat et al. 2002, 2005; Bell 2003; Kong et al. 2004; Burgarella et al. 2005; Calzetti et al. 2005; Seibert et al. 2005; Cortese et al. 2006; Schmitt et al. 2006; Iglesias-Páramo et al. 2006; Inoue et al. 2006). One consistent result relevant to the work presented here is that normal star-forming (nonstarburst) galaxies show larger scatter in plots of the infrared-to-ultraviolet ratio as a function of the ultraviolet spectral slope, with normal galaxies systematically exhibiting redder slopes than starburst galaxies. This broadening in the trend has been attributed to geometry, integrated versus local extractions, and/or the increased fractional 
TABLE 1

Galaxy Data

\begin{tabular}{|c|c|c|c|c|c|c|c|c|c|}
\hline Galaxy & Optical Morphology & $\begin{array}{c}\alpha_{0} \\
(\mathrm{~J} 2000.0)\end{array}$ & $\begin{array}{c}\delta_{0} \\
(\mathrm{~J} 2000.0)\end{array}$ & $\begin{array}{c}2 a \\
(\operatorname{arcsec})\end{array}$ & $\begin{array}{c}2 b \\
(\operatorname{arcsec})\end{array}$ & $\begin{array}{l}\text { P.A. } \\
\text { (deg) }\end{array}$ & $f_{\nu}(24)[$ unres $] / f_{\nu}(24)[\mathrm{res}]^{\mathrm{a}}$ & $f_{\nu}(24)[$ nuc $] / f_{\nu}(24)[\text { total }]^{\mathrm{a}}$ & $\begin{array}{c}\theta_{\mathrm{FUV}}{ }^{\mathrm{b}} \\
(\operatorname{arcsec})\end{array}$ \\
\hline NGC $0024^{\dagger} \ldots \ldots \ldots \ldots \ldots . . . .$. & SAc & 000955.9 & -245755 & 301 & 216 & 135 & 0.31 & 0.14 & 36.0 \\
\hline 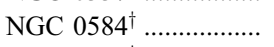 & $\mathrm{E} 4$ & 013120.6 & -065205 & 326 & 278 & 330 & 0.49 & 0.39 & 28.8 \\
\hline 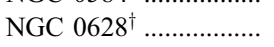 & SAc & 013641.8 & +154717 & 721 & 717 & 248 & 0.84 & 0.01 & 150. \\
\hline 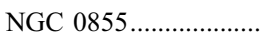 & $\mathrm{E}$ & 021403.9 & +275239 & 190 & 170 & 338 & 2.38 & 0.69 & $\ldots$ \\
\hline 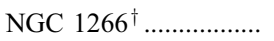 & SB0 & 031600.7 & -022541 & 234 & 232 & 0 & 9.09 & 0.87 & 10.5 \\
\hline NGC $1291^{\dagger} \ldots \ldots \ldots \ldots \ldots . . .$. & $\mathrm{SB} 0 / \mathrm{a}$ & 031719.1 & -410632 & 840 & 803 & 0 & 0.48 & 0.21 & 253 \\
\hline NGC $1316^{\dagger} \ldots \ldots \ldots \ldots \ldots \ldots$ & SAB0 & 032241.2 & -371210 & 864 & 583 & 230 & 0.60 & 0.05 & 89.2 \\
\hline 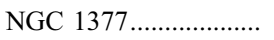 & S0 & 033639.0 & -205408 & 181 & 162 & 0 & $\sim 20$ & 0.85 & $\ldots$ \\
\hline 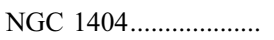 & E1 & 033852.3 & -353540 & 524 & 369 & 239 & 0.57 & 0.29 & $\ldots$ \\
\hline NGC $1482^{\dagger} \ldots \ldots \ldots \ldots . . . . .$. & SA0 & 035439.0 & -203009 & 349 & 310 & 29 & 5.26 & 0.77 & 19.0 \\
\hline M81 Dwarf A .................. & $\mathrm{I} ?$ & 082356.0 & +710145 & 78 & 78 & 0 & $\ldots$ & $\ldots$ & $\ldots$ \\
\hline 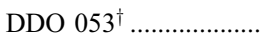 & $\operatorname{Im}$ & 083406.8 & +661036 & 133 & 110 & 30 & 7.69 & 0.08 & 22.9 \\
\hline NGC $2798^{\dagger} \ldots \ldots \ldots \ldots \ldots \ldots$ & $\mathrm{SBa}$ & 091723.1 & +415957 & 235 & 232 & 0 & $>10$ & 0.75 & 7.8 \\
\hline 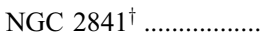 & $\mathrm{SAb}$ & 092203.3 & +505837 & 550 & 342 & 150 & 0.22 & 0.04 & 74.2 \\
\hline 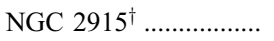 & I0 & 092609.4 & -763736 & 183 & 132 & 290 & 1.56 & 0.53 & $\ldots$ \\
\hline Holmberg $\mathrm{I}^{\dagger} \ldots \ldots \ldots \ldots \ldots . . . . .$. & $\mathrm{IABm}$ & 094030.5 & +711033 & 265 & 228 & 120 & 0.28 & 0.01 & 59.9 \\
\hline 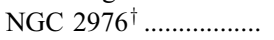 & SAc & 094715.3 & +675507 & 457 & 311 & 322 & 1.12 & 0.05 & 45.2 \\
\hline NGC $3049 \ldots \ldots \ldots \ldots \ldots$ & SBab & 095449.6 & +091614 & 218 & 160 & 119 & 5.88 & 0.74 & $\ldots$ \\
\hline 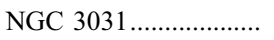 & SAab & 095531.8 & +690403 & 1628 & 1122 & 154 & 0.52 & 0.07 & 324 \\
\hline 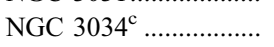 & I0 & $\ldots$ & .. & $\ldots$ & $\ldots$ & $\ldots$ & $\ldots$ & $\ldots$ & $\ldots$ \\
\hline Holmberg IX .................... & $\operatorname{Im}$ & 095729.2 & +690250 & 247 & 180 & 130 & $\ldots$ & $\ldots$ & 47.8 \\
\hline M81 Dwarf $\mathrm{B}^{\dagger} \ldots \ldots \ldots \ldots$ & $\operatorname{Im}$ & 100531.3 & +702152 & 107 & 69 & 140 & 1.61 & 0.50 & $\ldots$ \\
\hline 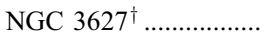 & $\mathrm{SABb}$ & 112013.4 & +125927 & 745 & 486 & 347 & 0.90 & 0.01 & 56.0 \\
\hline NGC $3773^{\dagger} \ldots \ldots \ldots \ldots . . . . . .$. & SA0 & 113813.1 & +120644 & 96 & 94 & 0 & 9.09 & 0.85 & $\ldots$ \\
\hline 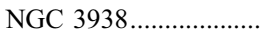 & SAc & 115250.3 & +440715 & 504 & 468 & 0 & 0.58 & 0.04 & $\ldots$ \\
\hline 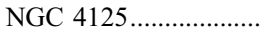 & E6p & 120805.8 & +651024 & 228 & 151 & 0 & 0.56 & 0.40 & $\ldots$ \\
\hline 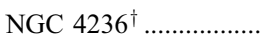 & SBdm & 121635.9 & +692808 & 1129 & 420 & 155 & 1.92 & 0.004 & 169 \\
\hline 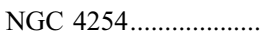 & $\mathrm{SAc}$ & 121849.7 & +142519 & 519 & 420 & 330 & 0.48 & 0.03 & $\ldots$ \\
\hline NGC 4321 ..................... & SABbc & 122254.8 & +154907 & 558 & 483 & 310 & 0.29 & 0.09 & $\ldots$ \\
\hline 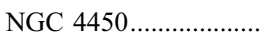 & SAab & 122830.1 & +170454 & 401 & 284 & 0 & 0.57 & 0.08 & $\ldots$ \\
\hline 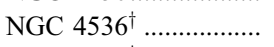 & SABbc & 123427.5 & +021113 & 454 & 376 & 30 & 3.45 & 0.48 & 79.4 \\
\hline 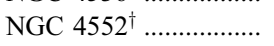 & E & 123539.8 & +123323 & 306 & 306 & 0 & 0.41 & 0.52 & 13.3 \\
\hline 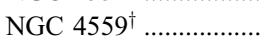 & SABcd & 123558.1 & +275752 & 576 & 327 & 50 & 0.83 & 0.04 & 87.1 \\
\hline 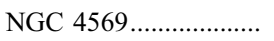 & SABab & 123650.2 & +131001 & 593 & 327 & 21 & 1.30 & 0.10 & 59.2 \\
\hline 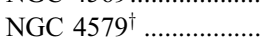 & $\mathrm{SABb}$ & 123743.6 & +114900 & 295 & 229 & 0 & 1.19 & 0.27 & 54.5 \\
\hline 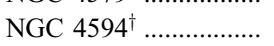 & SAa & 123959.4 & -113714 & 554 & 232 & 0 & 0.19 & 0.15 & 61.0 \\
\hline 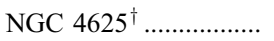 & SABmp & 124152.3 & +411618 & 198 & 190 & 140 & 0.55 & 0.21 & 28.1 \\
\hline 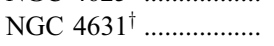 & SBd & 124203.7 & +323205 & 952 & 539 & 350 & 0.45 & 0.03 & 84.3 \\
\hline 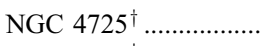 & SABab & 125027.7 & +252948 & 689 & 523 & 30 & 0.51 & 0.01 & 124 \\
\hline 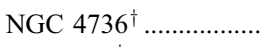 & SAab & 125056.7 & +410706 & 1033 & 824 & 10 & 0.65 & 0.07 & 40.7 \\
\hline 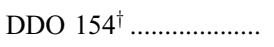 & $\mathrm{IBm}$ & 125405.2 & +270854 & 198 & 126 & 123 & 1.16 & 0.00 & 33.2 \\
\hline NGC 4826.................... & SAab & 125642.8 & +214050 & 722 & 448 & 112 & 0.32 & 0.14 & 41.2 \\
\hline DDO $165^{\dagger} \ldots \ldots \ldots \ldots \ldots$ & $\mathrm{Im}$ & 130625.0 & +674226 & 267 & 150 & 0 & $>10$ & 0.05 & 41.7 \\
\hline NGC 5033 .................... & SAc & 131328.2 & +363534 & 729 & 467 & 0 & 0.36 & 0.12 & $\ldots$ \\
\hline NGC 5055 .................... & $\mathrm{SAbc}$ & 131548.3 & +420142 & 893 & 682 & 11 & 0.38 & 0.04 & 108 \\
\hline NGC 5194 & $\mathrm{SABbc}$ & 132950.6 & +471307 & 1699 & 1129 & 285 & 0.51 & 0.002 & 143 \\
\hline 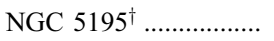 & SB0p & 132959.4 & +471556 & 202 & 191 & 0 & 3.32 & 0.61 & 110 \\
\hline
\end{tabular}


TABLE 1 - Continued

\begin{tabular}{|c|c|c|c|c|c|c|c|c|c|}
\hline Galaxy & Optical Morphology & $\begin{array}{c}\alpha_{0} \\
\text { (J2000.0) }\end{array}$ & $\begin{array}{c}\delta_{0} \\
(\mathrm{~J} 2000.0)\end{array}$ & $\begin{array}{c}2 a \\
(\operatorname{arcsec})\end{array}$ & $\begin{array}{c}2 b \\
(\operatorname{arcsec})\end{array}$ & $\begin{array}{l}\text { P.A. } \\
(\operatorname{deg})\end{array}$ & $f_{\nu}(24)[$ unres $] / f_{\nu}(24)[\text { res }]^{\mathrm{a}}$ & $f_{\nu}(24)[$ nuc $] / f_{\nu}(24)[\text { total }]^{\mathrm{a}}$ & $\begin{array}{c}\theta_{\mathrm{FUV}}^{\mathrm{b}} \\
(\operatorname{arcsec})\end{array}$ \\
\hline NGC $5474^{\dagger} \ldots \ldots \ldots \ldots \ldots . . .$. & SAcd & 140459.9 & +533913 & 386 & 335 & 120 & 0.68 & 0.04 & 84.3 \\
\hline NGC $5866^{\dagger} \ldots \ldots \ldots \ldots \ldots . . .$. & S0 & 150628.8 & +554551 & 500 & 306 & 39 & 1.41 & 0.34 & 26.3 \\
\hline IC 4710 & SBm & 182838.9 & -665903 & 313 & 219 & 30 & 1.89 & 0.03 & $\ldots$ \\
\hline NGC $6822^{\dagger} \ldots \ldots \ldots \ldots \ldots . . . .$. & $\mathrm{IBm}$ & 194453.2 & -144811 & 1453 & 1100 & 330 & 1.21 & 0.0005 & 257 \\
\hline NGC $7552^{\dagger} \ldots \ldots \ldots \ldots \ldots . .$. & SAc & 231610.8 & -423505 & 441 & 325 & 30 & 1.56 & 0.72 & 36.3 \\
\hline NGC $7793^{\dagger} \ldots \ldots \ldots \ldots \ldots . . . .$. & SAd & 235750.4 & -323530 & 754 & 498 & 0 & 0.67 & 0.03 & 109 \\
\hline
\end{tabular}

Notes.-Units of right ascension are hours, minutes, and seconds, and units of declination are degrees, arcminutes, and arcseconds. The ellipse parameters used in extracting optical and infrared fluxes are listed above. The position angle is measured east of north. Daggers indicate that the galaxy was used in the principal component analysis (see $\S 4.3$ ).

${ }^{a}$ See $\S$ 5.3. Entries are not included for NGC 3034 (saturated) and M81 Dwarf A and Holmberg IX (nondetections).

${ }^{\mathrm{b}}$ The equivalent radius of the ellipse including half of the total far-ultraviolet light.

c The bright core of NGC 3034 (M82) has rendered the Spitzer data extremely difficult to process. Saturation effects severely limit our ability to extract reliable flux densities.

contributions from recent (vs. current) star formation (e.g., Bell et al. 2002; Kong et al. 2004; Calzetti et al. 2005; Seibert et al. 2005; Boissier et al. 2007).

We are interested in exploring how the infrared-to-ultraviolet ratio depends on quantities like optical and mid-infrared morphology, ultraviolet and far-infrared color, and geometry within the Spitzer Nearby Galaxies Survey (SINGS) sample (Kennicutt et al. 2003). But in broader terms, the main focus of this paper is to simply present a panchromatic atlas of the broadband spectral energy distributions (SEDs) of a large, diverse sample of nearby galaxies and to quantify the variety of spectral shapes evident in such a sample. Since the fluxes presented in this work span wavelengths from the far-ultraviolet to the radio and are integrated over entire galaxies, this data set should prove useful to astronomers studying galaxies at high redshifts, where only information on the global properties of galaxies is accessible and the rest-frame ultraviolet data are shifted into optical bandpasses. One may plausibly argue that the variety of luminosities and spectral shapes typically seen in high-redshift surveys will be different than the diversity presented below for the SINGS sample (e.g., $10^{7} \lesssim L_{\mathrm{IR}} / L_{\odot} \lesssim 10^{11}$ ), since flux-limited surveys at high redshifts will mainly be sampling luminous and infrared-warm systems. On the other hand, deep far-infrared surveys show significant numbers of higher redshift systems similar to local normal star-forming galaxies in mass, size, and dust temperature (e.g., Chapman et al. 2002; Sajina et al. 2006). In either case, the rich collection of Spitzer, GALEX, and ancillary data provided by the SINGS project represents an important panchromatic baseline for extragalactic work.

Some of the analysis presented below could be accomplished using existing data sets, for example, the GALEX+UBV+2MASS+ IRAS work of Gil de Paz et al. (2007). However, the sensitivity and angular resolution of our Spitzer observations allow us to probe the dust emission in both bright and faint galaxies, and to do so in a spatially resolved manner. The paper is outlined as follows. In $\S 2$ we present the SINGS sample, while in $\S 3$ we present the collection of ultraviolet, optical, near-infrared, infrared, submillimeter, and radio data. The analysis of the broadband SEDs is described in $\S 4$, and the infrared-to-ultraviolet ratio is explored in detail in $\S 5$. A discussion and summary of the main results are provided in $\S 6$.

\section{THE SAMPLE}

The selection of the 75 galaxies in SINGS (Kennicutt et al. 2003) aimed to span a wide range in three key parameters (opti- cal morphology, luminosity, infrared-to-optical ratio) and to adequately sample several other secondary parameters (e.g., infrared color, metallicity, surface brightness, inclination, bar structure). The SINGS sample is comprised of nearby galaxies, with a median distance of $\sim 10 \mathrm{Mpc}$ and a maximum distance of $30 \mathrm{Mpc}$. SINGS galaxies come from a wide range of environments and galaxy types: low-metallicity dwarfs; quiescent elliptical galaxies; dusty grand-design spiral galaxies; Seyferts, LINERs, and star-forming nuclei of normal galaxies; systems within the Local Group and M81 group; and both field and (Virgo) cluster galaxies (Table 1).

\section{THE DATA}

Tables 2 and 3 present the global flux densities for the entire SINGS sample, for wavelengths spanning the ultraviolet through the radio. The data are corrected for Galactic extinction (Schlegel et al. 1998) assuming $A_{V} / E(B-V) \approx 3.1$ and the reddening curve of Li \& Draine (2001). The effect of air mass has been removed from the ground-based fluxes. Below follows a description of the new ultraviolet and optical and archival radio data collected for the SINGS program, in addition to a few updates to the Spitzer data presented in Dale et al. (2005).

\subsection{Ultraviolet Data}

The GALEX mission (Martin et al. 2005) is performing an allsky survey at ultraviolet wavelengths. The imaging portion of the survey is being carried out with a far-ultraviolet and a nearultraviolet filter centered at 1528 and $2271 \AA$ with respective FWHMs of 269 and $616 \AA$. In addition to imaging the entire sky with an effective exposure time of $\sim 0.1 \mathrm{ks}$, GALEX is also carrying out relatively deep integrations $(\sim 1.5 \mathrm{ks})$ for a few hundred nearby galaxies, including nearly the entire SINGS sample. With an angular resolution of $4^{\prime \prime}-6^{\prime \prime}$, the spatial details in GALEX images are well matched to those seen in Spitzer $24 \mu$ m imaging and more resolved than in Spitzer 70 and $160 \mu \mathrm{m}$ images. At the median distance of the SINGS sample ( 10 Mpc), the GALEX and MIPS $24 \mu \mathrm{m}$ data probe spatial scales of about $\sim 300 \mathrm{pc}$. This resolution, coupled with the GALEX field of view of $1.25^{\circ}$, allows for robust measures of sky-subtracted, spatially integrated ultraviolet fluxes even for large nearby galaxies.

Integrated ultraviolet fluxes are computed from the surface photometry profiles derived for the GALEX Atlas of Nearby Galaxies 
TABLE 2

Ultraviolet, Optical, and Near-Infrared Flux Densities

\begin{tabular}{|c|c|c|c|c|c|c|c|c|c|c|}
\hline Galaxy & $\begin{array}{c}E(B-V) \\
(\text { mag })\end{array}$ & $\begin{array}{c}\text { FUV } \\
1528 \AA \\
(\mathrm{mJy})\end{array}$ & $\begin{array}{c}\text { NUV } \\
2271 \AA \\
(\mathrm{mJy})\end{array}$ & $\begin{array}{c}B \\
0.45 \mu \mathrm{m} \\
(\mathrm{Jy})\end{array}$ & $\begin{array}{c}V \\
0.55 \mu \mathrm{m} \\
(\mathrm{Jy})\end{array}$ & $\begin{array}{c}R \\
0.66 \mu \mathrm{m} \\
(\mathrm{Jy})\end{array}$ & $\begin{array}{c}I \\
0.81 \mu \mathrm{m} \\
(\mathrm{Jy})\end{array}$ & $\begin{array}{c}J \\
1.25 \mu \mathrm{m} \\
(\mathrm{Jy})\end{array}$ & $\begin{array}{c}H \\
1.65 \mu \mathrm{m} \\
(\mathrm{Jy})\end{array}$ & $\begin{array}{c}K_{s} \\
2.17 \mu \mathrm{m} \\
(\mathrm{Jy})\end{array}$ \\
\hline NGC $0337 \ldots \ldots \ldots \ldots \ldots$ & 0.112 & $10.46 \pm 1.45$ & $18.69 \pm 2.59$ & 0.11 & 0.12 & 0.10 & 0.085 & 0.20 & 0.20 & 0.17 \\
\hline 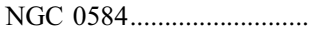 & 0.042 & $0.37 \pm 0.05$ & $2.00 \pm 0.28$ & 0.14 & 0.28 & 0.28 & 0.29 & 0.91 & 1.12 & 0.87 \\
\hline NGC 0628 & 0.070 & $75.96 \pm 10.52$ & $99.23 \pm 13.74$ & 0.65 & 0.84 & 0.76 & 0.65 & 1.66 & 1.67 & 1.32 \\
\hline NGC 1097............................ & 0.027 & $36.26 \pm 5.19$ & $50.97 \pm 7.18$ & 0.51 & 0.84 & 0.79 & 0.82 & 2.40 & 2.74 & 2.29 \\
\hline 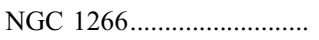 & 0.098 & $0.049 \pm 0.007$ & $0.29 \pm 0.04$ & 0.020 & 0.036 & 0.037 & 0.035 & 0.12 & 0.13 & 0.12 \\
\hline NGC $1291 \ldots \ldots \ldots$ & 0.013 & $7.38 \pm 1.02$ & $16.28 \pm 2.26$ & 0.76 & 1.48 & 1.37 & 1.48 & 4.34 & 4.48 & 3.93 \\
\hline 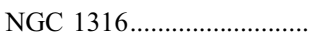 & 0.021 & $3.13 \pm 0.44$ & $16.58 \pm 2.30$ & 0.79 & 1.61 & 1.58 & 1.73 & 4.69 & 4.90 & 4.21 \\
\hline NGC $1377 \ldots \ldots \ldots$ & 0.028 & $\ldots$ & $\ldots$ & 0.012 & 0.023 & 0.021 & 0.033 & 0.10 & 0.11 & 0.095 \\
\hline NGC $1404 \ldots \ldots \ldots \ldots$ & 0.011 & $0.97 \pm 0.13$ & $2.76 \pm 0.38$ & 0.24 & 0.48 & 0.48 & 0.49 & 1.38 & 1.59 & 1.35 \\
\hline 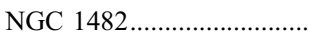 & 0.040 & $0.41 \pm 0.06$ & $1.43 \pm 0.21$ & 0.024 & 0.046 & 0.053 & 0.052 & 0.23 & 0.30 & 0.29 \\
\hline Holmberg II.............................. & 0.032 & $47.80 \pm 6.62$ & $48.23 \pm 6.68$ & 0.21 & 0.19 & 0.25 & 0.38 & 0.22 & 0.34 & 0.26 \\
\hline M81 Dwarf A ..................... & 0.020 & $0.48 \pm 0.07$ & $0.56 \pm 0.08$ & 0.002 & 0.001 & 0.001 & 0.002 & 0.004 & 0.004 & 0.003 \\
\hline DDO $053 \ldots \ldots$ & 0.038 & $2.65 \pm 0.37$ & $2.58 \pm 0.36$ & 0.011 & 0.008 & 0.006 & 0.007 & 0.008 & 0.014 & 0.008 \\
\hline NGC 2798 & 0.020 & $1.12 \pm 0.16$ & $2.33 \pm 0.32$ & 0.059 & 0.075 & 0.071 & 0.089 & 0.16 & 0.19 & 0.17 \\
\hline 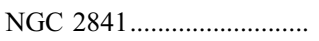 & 0.015 & $12.99 \pm 1.80$ & $20.57 \pm 2.85$ & 0.85 & 1.00 & 1.26 & 1.40 & 2.81 & 3.22 & 2.67 \\
\hline NGC 2915 ............................... & 0.275 & $16.13 \pm 2.23$ & $16.43 \pm 2.27$ & $0.077^{\mathrm{b}}$ & 0.069 & 0.071 & 0.077 & 0.13 & 0.15 & 0.092 \\
\hline 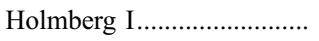 & 0.050 & $5.29 \pm 0.73$ & $5.60 \pm 0.78$ & 0.032 & 0.029 & 0.015 & 0.021 & 0.031 & 0.040 & 0.016 \\
\hline 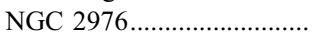 & 0.071 & $18.86 \pm 2.61$ & $30.24 \pm 4.19$ & 0.52 & 0.47 & 0.52 & 0.61 & 0.86 & 0.89 & 0.71 \\
\hline NGC $3049^{\mathrm{a}} \ldots \ldots \ldots \ldots \ldots \ldots$ & 0.038 & $\ldots$ & $4.51 \pm 0.62$ & 0.052 & 0.051 & 0.046 & 0.050 & 0.078 & 0.082 & 0.074 \\
\hline NGC $3031 \ldots \ldots \ldots \ldots$ & 0.080 & $178.9 \pm 24.8$ & $256.33 \pm 35.49$ & $5.07^{\mathrm{b}}$ & $8.73^{\mathrm{b}}$ & & & 23.47 & 25.44 & 21.29 \\
\hline 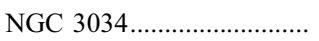 & 0.156 & $50.08 \pm 6.93$ & $105.3 \pm 14.6$ & 3.53 & $2.79^{\mathrm{b}}$ & 3.67 & 4.74 & 9.24 & 10.80 & 10.14 \\
\hline Holmberg IX & 0.079 & $4.01 \pm 0.56$ & $5.00 \pm 0.69$ & 0.014 & 0.010 & 0.008 & 0.010 & 0.025 & 0.021 & 0.015 \\
\hline 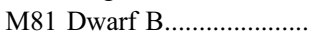 & 0.081 & $0.75 \pm 0.10$ & $0.92 \pm 0.13$ & 0.009 & 0.007 & 0.007 & 0.007 & 0.012 & 0.014 & 0.014 \\
\hline 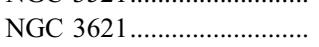 & 0.081 & $76.91 \pm 11.20$ & $110.2 \pm 15.8$ & $0.62^{\mathrm{b}}$ & 1.10 & $\ldots$ & 1.53 & 1.94 & 2.15 & 1.69 \\
\hline NGC $3627 \ldots \ldots \ldots$ & 0.033 & $30.46 \pm 4.22$ & $61.43 \pm 8.51$ & 1.51 & 1.63 & 1.51 & 1.90 & 3.34 & 3.73 & 3.17 \\
\hline NGC $3773 \ldots \ldots \ldots$ & 0.027 & $4.21 \pm 0.58$ & $5.55 \pm 0.77$ & 0.036 & 0.033 & 0.028 & 0.031 & 0.045 & 0.039 & 0.037 \\
\hline NGC $3938^{a}$ & 0.021 & $\ldots$ & $36.41 \pm 5.04$ & 0.44 & 0.44 & 0.34 & 0.41 & 0.64 & 0.58 & 0.54 \\
\hline NGC $4125^{\mathrm{a}} \ldots \ldots \ldots \ldots \ldots \ldots \ldots$ & 0.019 & $\ldots$ & $3.44 \pm 0.48$ & 0.49 & 0.54 & 0.66 & 0.87 & 1.39 & 1.54 & 1.29 \\
\hline NGC 4236 ................................. & 0.015 & $63.45 \pm 8.79$ & $76.24 \pm 10.56$ & 0.42 & 0.53 & 0.62 & 0.54 & 0.63 & 0.83 & 0.57 \\
\hline NGC $4254^{\mathrm{a}}$. & 0.039 & $\ldots$ & $61.82 \pm 8.56$ & 0.78 & 0.75 & 0.64 & 0.73 & 1.27 & 1.35 & 1.21 \\
\hline 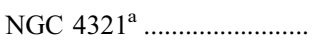 & 0.026 & $\ldots$ & $54.04 \pm 7.48$ & 0.50 & 0.70 & 0.85 & 1.23 & 1.87 & 2.00 & 1.65 \\
\hline NGC $4450^{\mathrm{a}} \ldots \ldots \ldots \ldots \ldots \ldots$ & 0.028 & $\ldots$ & $5.39 \pm 0.75$ & 0.43 & 0.53 & 0.52 & 0.65 & 1.20 & 1.39 & 1.08 \\
\hline NGC 4536 ............................... & 0.018 & $16.94 \pm 2.35$ & $21.93 \pm 3.04$ & 0.40 & 0.42 & 0.47 & 0.51 & 0.71 & 0.75 & 0.70 \\
\hline 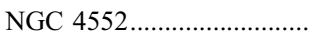 & 0.041 & $1.89 \pm 0.26$ & $4.66 \pm 0.65$ & 0.37 & 0.49 & 0.49 & 0.58 & 1.63 & 1.80 & 1.46 \\
\hline NGC $4559 \ldots \ldots \ldots \ldots \ldots$ & 0.018 & $53.79 \pm 7.45$ & $64.63 \pm 8.95$ & 0.66 & 0.50 & 0.50 & 0.58 & 0.77 & 0.79 & 0.66 \\
\hline NGC $4569 \ldots \ldots \ldots$ & 0.047 & $6.00 \pm 0.83$ & $19.69 \pm 2.73$ & $0.50^{\mathrm{b}}$ & $0.72^{\mathrm{b}}$ & $\ldots$ & $\ldots$ & 1.83 & 2.08 & 1.67 \\
\hline 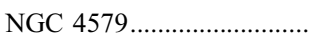 & 0.041 & $5.85 \pm 0.81$ & $12.11 \pm 1.68$ & 0.73 & 0.76 & 0.87 & 1.18 & 2.05 & 2.24 & 1.82 \\
\hline NGC 4594 & 0.051 & $5.55 \pm 0.77$ & $17.72 \pm 2.47$ & 2.25 & 2.76 & 3.41 & 4.30 & 8.06 & 9.19 & 7.57 \\
\hline NGC $4625 \ldots \ldots \ldots$ & 0.018 & $6.04 \pm 0.84$ & $7.97 \pm 1.10$ & 0.073 & 0.071 & 0.061 & 0.071 & 0.098 & 0.11 & 0.089 \\
\hline NGC $4631 \ldots \ldots \ldots \ldots$ & 0.017 & $80.95 \pm 11.21$ & $104.8 \pm 14.5$ & 1.19 & 0.91 & 0.96 & 1.12 & 1.75 & 1.98 & 1.84 \\
\hline 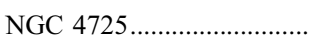 & 0.012 & $22.05 \pm 3.07$ & $29.61 \pm 4.13$ & 0.54 & 0.89 & 1.04 & 1.48 & 2.43 & 3.18 & 2.41 \\
\hline 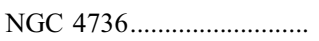 & 0.018 & $67.19 \pm 9.30$ & $91.87 \pm 12.72$ & 2.50 & 2.79 & 2.76 & 3.39 & 6.94 & 7.68 & 6.44 \\
\hline DDO $154 \ldots \ldots \ldots \ldots$ & 0.009 & $4.54 \pm 0.63$ & $4.42 \pm 0.61$ & 0.016 & 0.011 & 0.009 & 0.009 & 0.010 & 0.012 & 0.012 \\
\hline 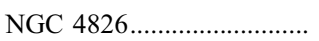 & 0.041 & $14.50 \pm 2.01$ & $37.45 \pm 5.19$ & 1.41 & 2.05 & $\ldots$ & $\ldots$ & 5.67 & 6.30 & 5.28 \\
\hline DDO $165 \ldots \ldots \ldots \ldots$ & 0.024 & $6.72 \pm 0.93$ & $8.15 \pm 1.13$ & 0.041 & 0.034 & 0.024 & 0.023 & 0.026 & 0.017 & 0.010 \\
\hline 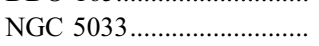 & 0.012 & 然 & $\ldots$ & 0.54 & 0.66 & $\ldots$ & 0.80 & 1.21 & 1.35 & 1.17 \\
\hline 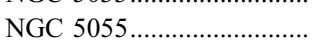 & 0.018 & $39.30 \pm 5.44$ & $63.42 \pm 8.78$ & $1.08^{\mathrm{b}}$ & $1.59^{\mathrm{b}}$ & $\cdots$ & $\ldots$ & 4.21 & 4.96 & 4.05 \\
\hline 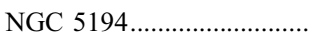 & 0.035 & $160.0 \pm 22.2$ & $260.8 \pm 36.1$ & 1.47 & 1.96 & 2.20 & 3.02 & 4.99 & 5.89 & 4.52 \\
\hline
\end{tabular}


TABLE 2-Continued

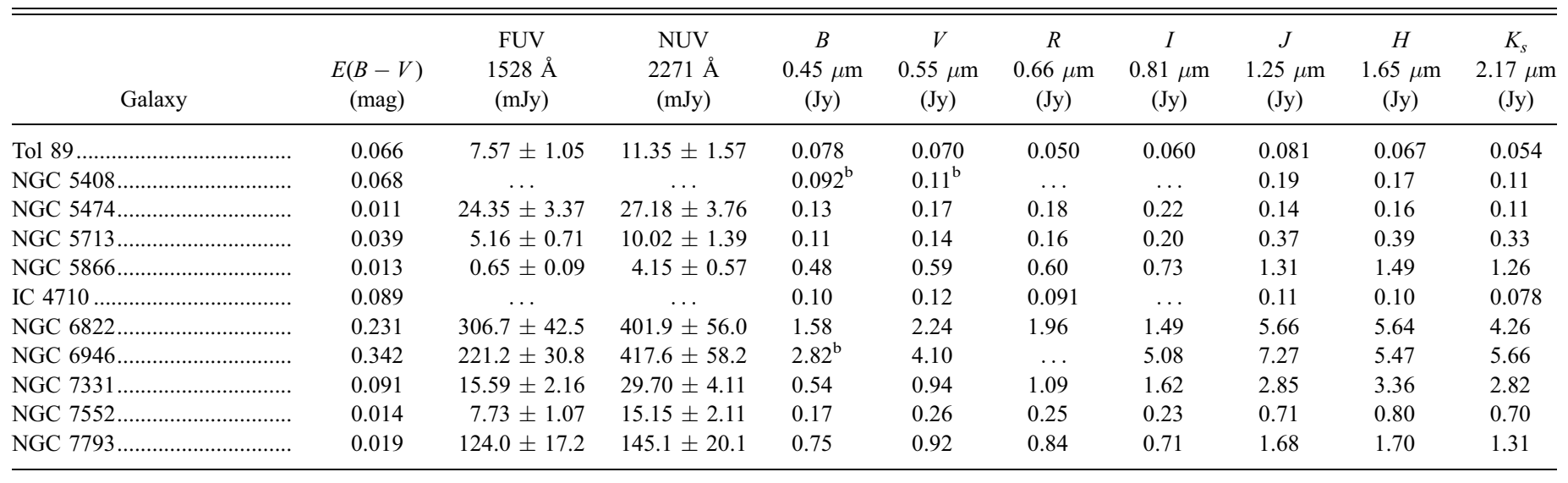

Notes.-See $\S 3$ for corrections that have been applied to the data. The uncertainties include both statistical and systematic effects ( $\lesssim 10 \%$ for the optical and nearinfrared data). The 2MASS near-infrared data are from Jarret et al. (2003).

${ }^{a}$ The far-ultraviolet detector was turned off during the observation.

${ }^{\mathrm{b}}$ Data from the RC3 catalog (de Vaucouleurs et al. 1991).

(Gil de Paz et al. 2007). ${ }^{18}$ Table 2 lists the global fluxes that include an asymptotic extrapolation to the isophotal profiles. The extrapolations are typically small and result in asymptotic fluxes that are, on average, $14 \%$ larger than those obtained at the optical radius: $\left\langle f_{\mathrm{UV}}\right.$ (asymptotic) $\left./ f_{\mathrm{UV}}\left(R_{25}\right)\right\rangle=1.14$ with a dispersion of 0.16 and 0.14 in the far- and near-ultraviolet, respectively. Foreground field stars and background galaxies were masked before flux extraction (see Gil de Paz et al. 2007). Some of the SINGS galaxies have not yet been observed with GALEX, but observations are soon planned (NGC 1377, NGC 3184, NGC 5033, and IC 4710), and a few only have near-ultraviolet observations because the far-ultraviolet detector was turned off at that time (see Table 2). Bright nearby stars make it unlikely that GALEX will obtain data for NGC 5408.

The uncertainties listed in Table 2 include the formal uncertainties from the weighted fits to the growth curves using the uncertainties of the individual points in the growth curves, in addition to absolute calibration uncertainties of $\sim 15 \%$ in both the far- and near-ultraviolet.

The average far-ultraviolet radiation field can be estimated from the far-ultraviolet fluxes and a beam size that characterizes the area from which the far-ultraviolet flux is emitted. In units of the local Milky Way field $\left(1.6 \times 10^{-3} \mathrm{ergs} \mathrm{cm}^{-2} \mathrm{~s}^{-1}\right)$, the average farultraviolet radiation field, uncorrected for extinction, can be expressed as

$$
G_{0}=2.2 \times 10^{3}\left[\frac{f_{\nu}(1528 \AA)}{\mathrm{mJy}}\right]\left(\frac{\operatorname{arcsec}}{\theta_{\mathrm{FUV}}}\right)^{2},
$$

where $\theta_{\mathrm{FUV}}$ is the equivalent radius of the ellipse including half of the total far-ultraviolet light (see Tables 1 and 2). Typical values for the SINGS sample span $1 \lesssim G_{0} \lesssim 25$ with a median value of $G_{0}=7.4$.

\subsection{Optical Data}

Although RC3 fluxes (de Vaucouleurs et al. 1991) in the $B$ and $V$ bands are available for a large portion of the SINGS sample, we pursued a $B V R I$ imaging campaign for reasons of consis-

\footnotetext{
${ }^{18}$ A few SINGS sources are not in the GALEX Atlas of Nearby Galaxies, but the observing and data reduction procedures for these galaxies are the same as for the Atlas targets (e.g., M81 Dwarf A, NGC 3773, NGC 4254, NGC 4725, NGC 6882, and NGC 6946).
}

tency, sensitivity, and completeness. The optical imaging for the SINGS project was carried out over the course of five observing runs at the Kitt Peak National Observatory (KPNO) $2.1 \mathrm{~m}$ telescope and one observing run at the Cerro Tololo Inter-American Observatory (CTIO) $1.5 \mathrm{~m}$ telescope between 2001 March and 2003 February. Broadband photometry was obtained in (Harris) $B V R I$ using $2 \mathrm{~K} \times 2 \mathrm{~K}$ CCDs with pixel scales and fields of view of $0.305^{\prime \prime}$ and $10^{\prime}$ at KPNO and $0.433^{\prime \prime}$ and $14.5^{\prime}$ at CTIO. Galaxies more extended than the $\mathrm{CCD}$ fields of view were imaged at multiple, overlapping pointings. Typical exposure times were $1440 \mathrm{~s}(B), 720 \mathrm{~s}(V), 420 \mathrm{~s}(R)$, and $840 \mathrm{~s}(I)$, usually split into two separate exposures to aid cosmic-ray removal. Such exposures reach a depth of about $25 \mathrm{mag} \operatorname{arcsec}^{-2}$ at a signal-to-noise ratio of $\sim 10$ per resolution element.

Data processing consisted of standard routines such as bias subtraction, flat-fielding with both dome and twilight flats, cosmicray removal, and the mosaicking of overlapping pointings for galaxies with large angular extents. The southern $3^{\prime}$ of the KPNO $2.1 \mathrm{~m} \mathrm{CCD}$ field of view suffers from vignetting; care is taken to remove as much of the vignetted portion of the KPNO images as feasible. Photometric standard stars were observed during each observing run to flux-calibrate the images. The images have photometric accuracy of $5 \%$ or better.

Global optical fluxes are extracted using the same apertures used for the IRAC and MIPS global flux extractions; these apertures cover at least the entire optical disk (see Table 1) and are chosen to be large enough to encompass all of the optical and infrared emission; in many instances the extended $160 \mu \mathrm{m}$ emission drives the final choice of aperture. Sky estimation and subtraction are carried out through the use of multiple sky apertures placed near the source without overlapping the faintest isophotes visible from the galaxy. Foreground stars are edited from the optical images after first being conservatively identified using $f_{\nu}(3.6 \mu \mathrm{m}) /$ $f_{\nu}(8.0 \mu \mathrm{m})$ and $f_{\nu}(8.0 \mu \mathrm{m}) / f_{\nu}(24 \mu \mathrm{m})$ color images [e.g., $f_{\nu}(8.0 \mu \mathrm{m}) / f_{\nu}(24 \mu \mathrm{m})>8$ for stars $]$.

\subsection{Infrared Data}

A full description of the infrared (2MASS, ISO, IRAS, Spitzer) and submillimeter (SCUBA) data can be found in Dale et al. (2005). In this section we present details of a few additional modifications and updates to the Spitzer data. For example, the MIPS flux calibrations and their uncertainties have been altered since Dale et al. (2005): the 24,70 , and $160 \mu \mathrm{m}$ calibration factors have 
TABLE 3

Infrared, Submillimeter, and Radio Flux Densities

\begin{tabular}{|c|c|c|c|c|c|c|c|c|c|c|}
\hline Galaxy & $\begin{array}{c}3.6 \mu \mathrm{m} \\
(\mathrm{Jy})\end{array}$ & $\begin{array}{c}4.5 \mu \mathrm{m} \\
\text { (Jy) }\end{array}$ & $\begin{array}{c}5.8 \mu \mathrm{m} \\
(\mathrm{Jy})\end{array}$ & $\begin{array}{c}8.0 \mu \mathrm{m} \\
(\mathrm{Jy})\end{array}$ & $\begin{array}{c}24 \mu \mathrm{m} \\
(\mathrm{Jy})\end{array}$ & $\begin{array}{c}70 \mu \mathrm{m} \\
(\mathrm{Jy})\end{array}$ & $\begin{array}{c}160 \mu \mathrm{m} \\
(\mathrm{Jy})\end{array}$ & $\begin{array}{c}450 \mu \mathrm{m} \\
(\mathrm{Jy})\end{array}$ & $\begin{array}{c}850 \mu \mathrm{m} \\
(\mathrm{Jy})\end{array}$ & $\begin{array}{l}20 \mathrm{~cm} \\
(\mathrm{mJy})\end{array}$ \\
\hline NGC $0024 \ldots \ldots \ldots \ldots \ldots \ldots$ & $0.10 \pm 0.01$ & $0.071 \pm 0.01$ & $0.089 \pm 0.01$ & $0.13 \pm 0.02$ & $0.14 \pm 0.007$ & $2.37 \pm 0.19$ & $8.19 \pm 1.05$ & $\ldots$ & & \\
\hline NGC 0337.............. & $0.097 \pm 0.01$ & $0.067 \pm 0.009$ & $0.14 \pm 0.02$ & $0.38 \pm 0.05$ & $0.68 \pm 0.03$ & $11.16 \pm 0.79$ & $20.09 \pm 2.44$ & $\ldots$ & $0.35 \pm 0.05$ & $110 \pm 11$ \\
\hline 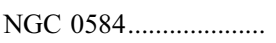 & $0.37 \pm 0.05$ & $0.22 \pm 0.03$ & $0.18 \pm 0.02$ & $0.11 \pm 0.01$ & $0.048 \pm 0.002$ & $0.18 \pm 0.05$ & $1.18 \pm 0.30^{\mathrm{a}}$ & $\ldots$ & $\ldots$ & $<50$ \\
\hline 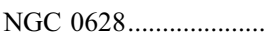 & $0.87 \pm 0.12$ & $0.54 \pm 0.08$ & $1.16 \pm 0.15$ & $2.70 \pm 0.34$ & $3.19 \pm 0.13$ & $34.78 \pm 2.50$ & $126.2 \pm 15.2$ & $\ldots$ & $\ldots$ & $173 \pm 17$ \\
\hline NGC $0855 \ldots \ldots \ldots \ldots$ & $0.043 \pm 0.006$ & $0.028 \pm 0.004$ & $0.019 \pm 0.003$ & $0.046 \pm 0.006$ & $0.087 \pm 0.004$ & $1.70 \pm 0.14$ & $2.50 \pm 0.36$ & $\ldots$ & $\ldots$ & $4.9 \pm 0.5$ \\
\hline 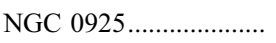 & $0.31 \pm 0.04$ & $0.21 \pm 0.03$ & $0.35 \pm 0.04$ & $0.61 \pm 0.08$ & $0.95 \pm 0.04$ & $14.40 \pm 1.04$ & $43.33 \pm 5.26$ & $\ldots$ & & $46 \pm 5$ \\
\hline NGC $1097 \ldots \ldots \ldots \ldots \ldots$ & $1.24 \pm 0.17$ & $0.80 \pm 0.11$ & $1.46 \pm 0.18$ & $3.19 \pm 0.40$ & $6.63 \pm 0.27$ & $59.84 \pm 4.66$ & $153.8 \pm 18.5$ & $\ldots$ & $1.44 \pm 0.78$ & $415 \pm 42$ \\
\hline NGC $1266 \ldots \ldots \ldots \ldots \ldots \ldots . . .$. & $0.055 \pm 0.008$ & $0.042 \pm 0.006$ & $0.057 \pm 0.008$ & $0.090 \pm 0.012$ & $0.88 \pm 0.04$ & $12.69 \pm 0.95$ & $10.30 \pm 1.29$ & $\ldots$ & $\ldots$ & $116 \pm 12$ \\
\hline 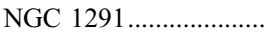 & $2.11 \pm 0.29$ & $1.27 \pm 0.17$ & $0.96 \pm 0.12$ & $0.64 \pm 0.08$ & $0.57 \pm 0.02$ & $6.29 \pm 0.46$ & $28.60 \pm 3.49$ & $\ldots$ & $\ldots$ & $\ldots$ \\
\hline 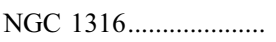 & $2.48 \pm 0.34$ & $1.53 \pm 0.21$ & $1.13 \pm 0.14$ & $0.55 \pm 0.07$ & $0.43 \pm 0.02$ & $5.44 \pm 0.40$ & $12.61 \pm 1.78$ & $\ldots$ & $\ldots$ & $256 \pm 26$ \\
\hline NGC 1377 ............... & $0.057 \pm 0.008$ & $0.085 \pm 0.012$ & $0.27 \pm 0.04$ & $0.42 \pm 0.05$ & $1.83 \pm 0.08$ & $6.35 \pm 0.47$ & $3.38 \pm 0.42$ & $\ldots$ & $\ldots$ & $<1.0$ \\
\hline NGC $1404 \ldots \ldots \ldots \ldots$ & $0.73 \pm 0.10$ & $0.43 \pm 0.06$ & $0.33 \pm 0.04$ & $0.16 \pm 0.02$ & $0.088 \pm 0.004$ & $0.17 \pm 0.12^{\mathrm{a}}$ & $0.29 \pm 0.28^{\mathrm{a}}$ & $\ldots$ & $\ldots$ & $3.9 \pm 0.6$ \\
\hline NGC $1482 \ldots \ldots \ldots \ldots \ldots \ldots$ & $0.21 \pm 0.03$ & $0.15 \pm 0.02$ & $0.59 \pm 0.08$ & $1.56 \pm 0.19$ & $3.69 \pm 0.15$ & $32.45 \pm 2.88$ & $38.79 \pm 4.69$ & $\ldots$ & $0.33 \pm 0.05$ & $239 \pm 24$ \\
\hline NGC $1512 \ldots \ldots \ldots \ldots \ldots$ & $0.39 \pm 0.05$ & $0.24 \pm 0.03$ & $0.27 \pm 0.03$ & $0.44 \pm 0.05$ & $0.46 \pm 0.02$ & $6.65 \pm 0.48$ & $23.70 \pm 2.86$ & $\ldots$ & $\ldots$ & $7.0 \pm 1$ \\
\hline NGC 1566...................... & $0.75 \pm 0.10$ & $0.48 \pm 0.07$ & $0.91 \pm 0.12$ & $2.11 \pm 0.26$ & $2.83 \pm 0.13$ & $34.32 \pm 2.51$ & $102.1 \pm 12.3$ & $\ldots$ & & $400 \pm 40$ \\
\hline NGC $1705 \ldots \ldots \ldots \ldots$ & $0.026 \pm 0.004$ & $0.018 \pm 0.003$ & $0.010 \pm 0.002$ & $0.017 \pm 0.002$ & $0.056 \pm 0.002$ & $1.38 \pm 0.10$ & $1.66 \pm 0.21$ & $\ldots$ & $\ldots$ & \\
\hline NGC $2403 \ldots \ldots \ldots \ldots \ldots \ldots$ & $1.88 \pm 0.25$ & $1.31 \pm 0.18$ & $2.13 \pm 0.27$ & $4.11 \pm 0.51$ & $5.84 \pm 0.24$ & $86.36 \pm 6.18$ & $245.6 \pm 29.6$ & $\ldots$ & $\ldots$ & $330 \pm 33$ \\
\hline Holmberg II..................... & $0.071 \pm 0.010$ & $0.057 \pm 0.008$ & $0.031 \pm 0.005$ & $0.024 \pm 0.005$ & $0.20 \pm 0.008$ & $3.67 \pm 0.26$ & $4.46 \pm 0.58$ & & $\ldots$ & $20 \pm 3$ \\
\hline M81 Dwarf A ................... & $0.002 \pm 0.001$ & $0.001 \pm 0.001$ & $<0.004$ & $<0.002$ & $<0.018$ & $<0.17$ & $<0.15$ & $\ldots$ & $\ldots$ & $\ldots$ \\
\hline DDO 053 & $0.005 \pm 0.001$ & $0.004 \pm 0.001$ & $0.003 \pm 0.001$ & $0.007 \pm 0.001$ & $0.029 \pm 0.001$ & $0.40 \pm 0.03$ & $0.50 \pm 0.11$ & $\ldots$ & $\ldots$ & $\ldots$ \\
\hline NGC $2798 \ldots \ldots \ldots \ldots \ldots$ & $0.11 \pm 0.02$ & $0.081 \pm 0.011$ & $0.27 \pm 0.03$ & $0.63 \pm 0.08$ & $2.62 \pm 0.11$ & $21.72 \pm 1.79$ & $20.69 \pm 2.50$ & $\ldots$ & $0.19 \pm 0.03$ & $83 \pm 9$ \\
\hline NGC $2841 \ldots \ldots \ldots \ldots \ldots \ldots$ & $1.27 \pm 0.17$ & $0.75 \pm 0.10$ & $0.67 \pm 0.09$ & $1.16 \pm 0.14$ & $0.91 \pm 0.04$ & $10.22 \pm 0.73$ & $62.29 \pm 7.54$ & $\ldots$ & $\ldots$ & $84 \pm 9$ \\
\hline NGC $2915 \ldots \ldots \ldots \ldots \ldots$ & $0.054 \pm 0.008$ & $0.035 \pm 0.005$ & $0.033 \pm 0.004$ & $0.031 \pm 0.004$ & $0.063 \pm 0.003$ & $1.41 \pm 0.11$ & $1.46 \pm 0.27$ & $\ldots$ & $\ldots$ & $\ldots$ \\
\hline Holmberg I........................ & $0.012 \pm 0.001$ & $0.008 \pm 0.001$ & $0.007 \pm 0.002$ & $0.008 \pm 0.002$ & $0.013 \pm 0.002$ & $0.42 \pm 0.08$ & $0.90 \pm 0.17$ & $\ldots$ & & $\ldots$ \\
\hline 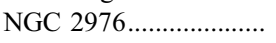 & $0.43 \pm 0.06$ & $0.28 \pm 0.04$ & $0.51 \pm 0.07$ & $1.02 \pm 0.13$ & $1.37 \pm 0.06$ & $20.43 \pm 1.45$ & $52.56 \pm 6.35$ & $\ldots$ & $0.61 \pm 0.24$ & $51 \pm 5$ \\
\hline NGC $3049 \ldots \ldots \ldots \ldots \ldots \ldots$ & $0.040 \pm 0.005$ & $0.028 \pm 0.004$ & $0.065 \pm 0.009$ & $0.14 \pm 0.02$ & $0.43 \pm 0.02$ & $2.90 \pm 0.21$ & $4.86 \pm 0.59$ & $\ldots$ & $\ldots$ & $12 \pm 2$ \\
\hline 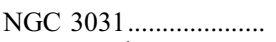 & $10.92 \pm 1.48$ & $6.53 \pm 0.90$ & $5.96 \pm 0.75$ & $8.04 \pm 1.00$ & $5.09 \pm 0.20$ & $85.18 \pm 5.96$ & $360.0 \pm 43.4$ & & $\ldots$ & $380 \pm 38$ \\
\hline NGC $3034^{\mathrm{b}} \ldots \ldots \ldots \ldots \ldots \ldots$ & & & & & & & & $39.2 \pm 9.8$ & $5.51 \pm 0.83$ & $7660 \pm 770$ \\
\hline Holmberg IX ...................... & $0.007 \pm 0.001$ & $0.004 \pm 0.001$ & $<0.013$ & $<0.012$ & $<0.037$ & $<0.25$ & $<0.48$ & $\ldots$ & $\ldots$ & $\ldots$ \\
\hline M81 Dwarf B............... & $0.005 \pm 0.001$ & $0.004 \pm 0.001$ & $0.003 \pm 0.001$ & $0.003 \pm 0.001$ & $0.009 \pm 0.001$ & $0.15 \pm 0.03$ & $0.39 \pm 0.18$ & $\ldots$ & $\ldots$ & $\ldots$ \\
\hline NGC $3190 \ldots \ldots \ldots \ldots \ldots \ldots$ & $0.37 \pm 0.05$ & $0.24 \pm 0.03$ & $0.25 \pm 0.03$ & $0.33 \pm 0.04$ & $0.27 \pm 0.01$ & $5.66 \pm 0.40$ & $15.01 \pm 1.82$ & $\ldots$ & $0.19 \pm 0.04$ & $43 \pm 5$ \\
\hline 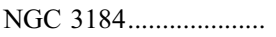 & $0.56 \pm 0.08$ & $0.36 \pm 0.05$ & $0.67 \pm 0.08$ & $1.44 \pm 0.18$ & $1.43 \pm 0.06$ & $15.76 \pm 1.12$ & $70.48 \pm 8.50$ & $\ldots$ & $\ldots$ & $56 \pm 5$ \\
\hline NGC 3198........................... & $0.27 \pm 0.04$ & $0.17 \pm 0.02$ & $0.34 \pm 0.04$ & $0.68 \pm 0.09$ & $1.06 \pm 0.04$ & $10.27 \pm 0.73$ & $39.00 \pm 4.93$ & $\ldots$ & $\ldots$ & $27 \pm 3$ \\
\hline IC 2574 & $0.15 \pm 0.02$ & $0.091 \pm 0.013$ & $0.066 \pm 0.009$ & $0.066 \pm 0.009$ & $0.28 \pm 0.01$ & $5.55 \pm 0.43$ & $11.75 \pm 1.50$ & $\ldots$ & $\ldots$ & $11 \pm 2$ \\
\hline 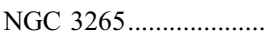 & $0.028 \pm 0.004$ & $0.020 \pm 0.003$ & $0.041 \pm 0.005$ & $0.10 \pm 0.01$ & $0.30 \pm 0.01$ & $2.71 \pm 0.20$ & $2.70 \pm 0.34$ & $\ldots$ & $\ldots$ & $11 \pm 2$ \\
\hline Mrk 33 ......................... & $0.027 \pm 0.004$ & $0.019 \pm 0.003$ & $0.053 \pm 0.007$ & $0.13 \pm 0.02$ & $0.86 \pm 0.04$ & $4.35 \pm 0.32$ & $3.87 \pm 0.48$ & $\ldots$ & $0.04 \pm 0.01$ & $17 \pm 2$ \\
\hline NGC $3351 \ldots \ldots \ldots \ldots$ & $0.81 \pm 0.11$ & $0.51 \pm 0.07$ & $0.73 \pm 0.09$ & $1.33 \pm 0.16$ & $2.58 \pm 0.12$ & $24.18 \pm 1.87$ & $67.49 \pm 8.28$ & $\ldots$ & & $44 \pm 5$ \\
\hline 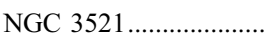 & $2.05 \pm 0.28$ & $1.36 \pm 0.19$ & $2.56 \pm 0.32$ & $6.27 \pm 0.76$ & $5.51 \pm 0.22$ & $63.13 \pm 4.54$ & $222.3 \pm 26.8$ & $\ldots$ & $2.11 \pm 0.82$ & $357 \pm 36$ \\
\hline 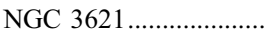 & $0.99 \pm 0.13$ & $0.67 \pm 0.09$ & $1.62 \pm 0.21$ & $3.51 \pm 0.44$ & $3.70 \pm 0.19$ & $50.21 \pm 3.94$ & $139.0 \pm 17.1$ & $\ldots$ & $\ldots$ & $198 \pm 20$ \\
\hline NGC $3627 \ldots \ldots \ldots \ldots \ldots$ & $1.87 \pm 0.25$ & $1.25 \pm 0.17$ & $2.39 \pm 0.30$ & $5.58 \pm 0.69$ & $7.42 \pm 0.30$ & $92.63 \pm 7.00$ & $230.2 \pm 27.7$ & $\ldots$ & $1.86 \pm 0.70$ & $458 \pm 46$ \\
\hline NGC $3773 \ldots \ldots \ldots \ldots \ldots$ & $0.022 \pm 0.003$ & $0.014 \pm 0.002$ & $0.026 \pm 0.004$ & $0.048 \pm 0.006$ & $0.14 \pm 0.006$ & $1.58 \pm 0.12$ & $2.38 \pm 0.33$ & $\ldots$ & $\ldots$ & $5.8 \pm 0.5$ \\
\hline 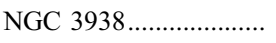 & $0.32 \pm 0.04$ & $0.21 \pm 0.03$ & $0.41 \pm 0.05$ & $0.98 \pm 0.12$ & $1.09 \pm 0.04$ & $14.25 \pm 1.01$ & $51.98 \pm 6.26$ & $\ldots$ & $\ldots$ & $62 \pm 7$ \\
\hline NGC 4125 ....................... & $0.64 \pm 0.09$ & $0.37 \pm 0.05$ & $0.25 \pm 0.03$ & $0.14 \pm 0.02$ & $0.079 \pm 0.004$ & $1.11 \pm 0.10$ & $1.77 \pm 0.28$ & & $\ldots$ & $<50$ \\
\hline NGC 4236.................... & $0.25 \pm 0.03$ & $0.21 \pm 0.03$ & $0.11 \pm 0.01$ & $0.22 \pm 0.03$ & $0.55 \pm 0.02$ & $8.27 \pm 0.59$ & $20.43 \pm 2.52$ & $\ldots$ & $\ldots$ & $28 \pm 3$ \\
\hline NGC 4254..................... & $0.70 \pm 0.10$ & $0.47 \pm 0.06$ & $1.49 \pm 0.19$ & $3.94 \pm 0.49$ & $4.20 \pm 0.17$ & $50.29 \pm 3.60$ & $142.9 \pm 17.2$ & $\ldots$ & $1.01 \pm 0.54$ & $422 \pm 42$ \\
\hline NGC $4321 \ldots \ldots \ldots \ldots$ & $0.95 \pm 0.13$ & $0.64 \pm 0.09$ & $1.22 \pm 0.15$ & $2.89 \pm 0.36$ & $3.34 \pm 0.13$ & $40.59 \pm 2.90$ & $139.6 \pm 16.8$ & $\ldots$ & $0.88 \pm 0.49$ & $340 \pm 34$ \\
\hline NGC 4450..................... & $0.53 \pm 0.07$ & $0.33 \pm 0.04$ & $0.26 \pm 0.03$ & $0.27 \pm 0.03$ & $0.21 \pm 0.01$ & $3.42 \pm 0.29$ & $16.94 \pm 2.14$ & $\ldots$ & $\ldots$ & $9.4 \pm 1$ \\
\hline
\end{tabular}


TABLE 3-Continued

\begin{tabular}{|c|c|c|c|c|c|c|c|c|c|c|}
\hline Galaxy & $\begin{array}{c}3.6 \mu \mathrm{m} \\
(\mathrm{Jy})\end{array}$ & $\begin{array}{c}4.5 \mu \mathrm{m} \\
(\mathrm{Jy})\end{array}$ & $\begin{array}{c}5.8 \mu \mathrm{m} \\
(\mathrm{Jy})\end{array}$ & $\begin{array}{c}8.0 \mu \mathrm{m} \\
(\mathrm{Jy})\end{array}$ & $\begin{array}{c}24 \mu \mathrm{m} \\
(\mathrm{Jy})\end{array}$ & $\begin{array}{c}70 \mu \mathrm{m} \\
(\mathrm{Jy})\end{array}$ & $\begin{array}{c}160 \mu \mathrm{m} \\
(\mathrm{Jy})\end{array}$ & $\begin{array}{c}450 \mu \mathrm{m} \\
(\mathrm{Jy})\end{array}$ & $\begin{array}{c}850 \mu \mathrm{m} \\
(\mathrm{Jy})\end{array}$ & $\begin{array}{l}20 \mathrm{~cm} \\
(\mathrm{mJy})\end{array}$ \\
\hline NGC $4536 \ldots \ldots \ldots \ldots \ldots$. & $0.40 \pm 0.05$ & $0.29 \pm 0.04$ & $0.62 \pm 0.08$ & $1.66 \pm 0.21$ & $3.46 \pm 0.14$ & $31.99 \pm 2.49$ & $58.09 \pm 7.00$ & $\ldots$ & $0.42 \pm 0.11$ & $194 \pm 19$ \\
\hline NGC 4552 ..................... & $0.83 \pm 0.11$ & $0.49 \pm 0.07$ & $0.32 \pm 0.04$ & $0.17 \pm 0.02$ & $0.094 \pm 0.004$ & $0.54 \pm 0.11$ & $1.42 \pm 0.73$ & $\ldots$ & $\ldots$ & $100 \pm 3$ \\
\hline NGC $4559 . . . \ldots \ldots \ldots \ldots . . . . . . . . .$. & $0.35 \pm 0.05$ & $0.23 \pm 0.03$ & $0.42 \pm 0.05$ & $0.84 \pm 0.10$ & $1.12 \pm 0.05$ & $16.89 \pm 1.20$ & $54.15 \pm 6.53$ & $\ldots$ & & $65 \pm 7$ \\
\hline 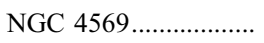 & $0.76 \pm 0.10$ & $0.47 \pm 0.06$ & $0.59 \pm 0.08$ & $1.02 \pm 0.13$ & $1.44 \pm 0.06$ & $12.37 \pm 0.88$ & $41.21 \pm 5.17$ & $\ldots$ & $0.47 \pm 0.08$ & $83 \pm 9$ \\
\hline 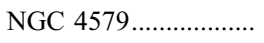 & $0.87 \pm 0.12$ & $0.52 \pm 0.07$ & $0.54 \pm 0.07$ & $0.73 \pm 0.09$ & $0.76 \pm 0.03$ & $9.53 \pm 0.75$ & $41.03 \pm 4.95$ & $\ldots$ & $0.44 \pm 0.07$ & $98 \pm 10$ \\
\hline 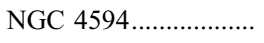 & $3.94 \pm 0.53$ & $2.31 \pm 0.32$ & $1.75 \pm 0.22$ & $1.30 \pm 0.16$ & $0.71 \pm 0.04$ & $8.02 \pm 0.68$ & $42.12 \pm 5.58$ & $\ldots$ & $0.37 \pm 0.11$ & $137 \pm 14$ \\
\hline NGC $4625 \ldots \ldots \ldots \ldots . . .$. & $0.049 \pm 0.006$ & $0.030 \pm 0.004$ & $0.059 \pm 0.008$ & $0.13 \pm 0.02$ & $0.13 \pm 0.006$ & $2.06 \pm 0.16$ & $5.42 \pm 0.68$ & $\ldots$ & & $7.1 \pm 2$ \\
\hline 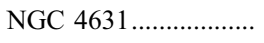 & $1.26 \pm 0.17$ & $0.84 \pm 0.11$ & $2.49 \pm 0.31$ & $5.86 \pm 0.73$ & $8.15 \pm 0.33$ & $130.2 \pm 9.9$ & $289.5 \pm 34.9$ & $30.7 \pm 10.0$ & $5.73 \pm 1.21$ & $1200 \pm 120$ \\
\hline NGC $4725 \ldots \ldots \ldots \ldots \ldots$ & $1.14 \pm 0.15$ & $0.70 \pm 0.10$ & $0.75 \pm 0.10$ & $1.21 \pm 0.15$ & $0.86 \pm 0.04$ & $8.85 \pm 0.66$ & $59.91 \pm 7.36$ & $\ldots$ & & $28 \pm 3$ \\
\hline NGC $4736 \ldots \ldots \ldots \ldots . . .$. & $3.60 \pm 0.49$ & $2.32 \pm 0.32$ & $2.76 \pm 0.35$ & $5.17 \pm 0.64$ & $5.65 \pm 0.23$ & $93.93 \pm 7.34$ & $177.4 \pm 21.4$ & $\ldots$ & $1.54 \pm 0.66$ & $271 \pm 27$ \\
\hline 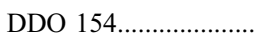 & $0.004 \pm 0.001$ & $0.003 \pm 0.001$ & $<0.006$ & $<0.004$ & $0.008 \pm 0.001^{\mathrm{a}}$ & $0.065 \pm 0.05^{\mathrm{a}}$ & $0.35 \pm 0.12^{\mathrm{a}}$ & $\ldots$ & & \\
\hline 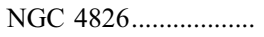 & $2.52 \pm 0.34$ & $1.57 \pm 0.22$ & $1.66 \pm 0.21$ & $2.35 \pm 0.29$ & $2.72 \pm 0.15$ & $55.16 \pm 5.05$ & $98.82 \pm 12.67$ & $\ldots$ & $1.23 \pm 0.31$ & $101 \pm 10$ \\
\hline 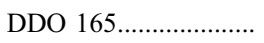 & $0.016 \pm 0.002$ & $0.012 \pm 0.002$ & $0.005 \pm 0.002$ & $0.004 \pm 0.001^{\mathrm{a}}$ & $0.014 \pm 0.001^{\mathrm{a}}$ & $0.15 \pm 0.07^{\mathrm{a}}$ & $0.33 \pm 0.26^{\mathrm{a}}$ & $\ldots$ & & \\
\hline NGC 5033.................... & $0.64 \pm 0.09$ & $0.47 \pm 0.06$ & $0.82 \pm 0.10$ & $1.92 \pm 0.24$ & $1.97 \pm 0.08$ & $28.81 \pm 2.09$ & $91.07 \pm 11.2$ & $\ldots$ & $1.10 \pm 0.55$ & $178 \pm 18$ \\
\hline 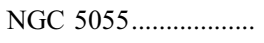 & $2.38 \pm 0.32$ & $1.55 \pm 0.21$ & $2.67 \pm 0.34$ & $5.64 \pm 0.70$ & $5.73 \pm 0.23$ & $72.57 \pm 5.16$ & $302.3 \pm 36.6$ & $\ldots$ & & $390 \pm 39$ \\
\hline NGC 5194..................... & $2.66 \pm 0.36$ & $1.80 \pm 0.25$ & $4.29 \pm 0.54$ & $10.64 \pm 1.32$ & $12.67 \pm 0.53$ & $147.1 \pm 10.6$ & $494.7 \pm 59.8$ & $\ldots$ & $2.61 \pm 0.39$ & $1490 \pm 150$ \\
\hline NGC $5195 \ldots \ldots \ldots \ldots \ldots$ & $0.83 \pm 0.11$ & $0.51 \pm 0.07$ & $0.47 \pm 0.06$ & $0.65 \pm 0.08$ & $1.40 \pm 0.27$ & $16.31 \pm 3.26$ & $14.86 \pm 2.97$ & $\ldots$ & $0.26 \pm 0.04$ & $50 \pm 5$ \\
\hline 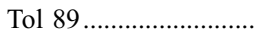 & $0.038 \pm 0.005$ & $0.025 \pm 0.004$ & $0.014 \pm 0.002$ & $0.059 \pm 0.008$ & $0.28 \pm 0.01$ & $2.03 \pm 0.16$ & $3.52 \pm 0.51$ & $\ldots$ & & $4.2 \pm 0.8$ \\
\hline NGC 5408 ................... & $0.052 \pm 0.007$ & $0.037 \pm 0.005$ & $0.041 \pm 0.005$ & $0.038 \pm 0.005$ & $0.43 \pm 0.02$ & $3.59 \pm 0.27$ & $2.57 \pm 0.38$ & $\ldots$ & & \\
\hline NGC 5474 .................... & $0.10 \pm 0.01$ & $0.073 \pm 0.010$ & $0.077 \pm 0.010$ & $0.12 \pm 0.01$ & $0.18 \pm 0.008$ & $3.73 \pm 0.27$ & $10.56 \pm 1.29$ & $\ldots$ & & $12 \pm 2$ \\
\hline NGC $5713 \ldots \ldots \ldots \ldots$ & $0.20 \pm 0.03$ & $0.14 \pm 0.02$ & $0.30 \pm 0.04$ & $1.16 \pm 0.15$ & $2.35 \pm 0.10$ & $23.69 \pm 1.84$ & $39.66 \pm 4.79$ & & $0.57 \pm 0.12$ & $160 \pm 16$ \\
\hline NGC 5866.................. & $0.66 \pm 0.09$ & $0.42 \pm 0.06$ & $0.31 \pm 0.04$ & $0.31 \pm 0.04$ & $0.21 \pm 0.009$ & $8.71 \pm 0.63$ & $17.74 \pm 2.14$ & $0.8 \pm 0.2$ & $0.14 \pm 0.02$ & $23 \pm 3$ \\
\hline 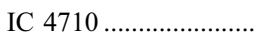 & $0.070 \pm 0.010$ & $0.047 \pm 0.007$ & $0.045 \pm 0.006$ & $0.065 \pm 0.008$ & $0.12 \pm 0.005$ & $2.37 \pm 0.18$ & $3.57 \pm 0.48$ & $\ldots$ & & $\ldots$ \\
\hline 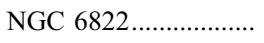 & $2.12 \pm 0.29$ & $1.38 \pm 0.19$ & $1.45 \pm 0.18$ & $1.41 \pm 0.18$ & $3.18 \pm 0.13$ & $63.75 \pm 4.50$ & $143.5 \pm 17.4$ & $\ldots$ & & $69 \pm 14$ \\
\hline 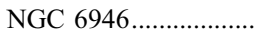 & $3.31 \pm 0.45$ & $2.18 \pm 0.30$ & $5.88 \pm 0.74$ & $14.12 \pm 1.76$ & $20.37 \pm 0.81$ & $207.2 \pm 16.1$ & $502.8 \pm 60.6$ & $18.5 \pm 4.6$ & $2.98 \pm 0.45$ & $1395 \pm 140$ \\
\hline NGC $7331 \ldots \ldots \ldots \ldots \ldots$ & $1.61 \pm 0.22$ & $1.02 \pm 0.14$ & $1.87 \pm 0.24$ & $4.05 \pm 0.50$ & $4.36 \pm 0.25$ & $74.97 \pm 6.62$ & $189.5 \pm 24.3$ & $20.6 \pm 8.1$ & $2.11 \pm 0.38$ & $373 \pm 37$ \\
\hline NGC $7552 \ldots \ldots \ldots \ldots \ldots$ & $0.45 \pm 0.06$ & $0.36 \pm 0.05$ & $1.07 \pm 0.14$ & $2.71 \pm 0.34$ & $10.66 \pm 0.44^{\mathrm{c}}$ & $67.59 \pm 11.1^{\mathrm{c}}$ & $93.39 \pm 11.25$ & $\ldots$ & $0.80 \pm 0.17$ & $276 \pm 28$ \\
\hline NGC $7793 \ldots \ldots \ldots . . . . . . .$. & $0.77 \pm 0.10$ & $0.47 \pm 0.06$ & $1.04 \pm 0.13$ & $1.85 \pm 0.23$ & $2.05 \pm 0.08$ & $34.29 \pm 2.43$ & $126.2 \pm 15.3$ & $\ldots$ & & $103 \pm 10$ \\
\hline
\end{tabular}

Notes.-See $\S 3$ for details on the data. Upper limits $(3 \sigma)$ are provided for nondetections.

a Possibly severely contaminated by background source(s).

${ }^{b}$ The bright core of NGC 3034 (M82) has rendered the Spitzer data extremely difficult to process. Saturation effects severely limit our ability to extract reliable global flux densities.

${ }^{\mathrm{c}}$ Flux artificially low due to saturation effects. 
TABLE 4

IRAC Aperture Correction Parameters

\begin{tabular}{|c|c|c|c|}
\hline$\lambda$ & $A$ & $B$ & $C$ \\
\hline $3.5 \mu \mathrm{m}$ & 0.82 & 0.370 & 0.910 \\
\hline $4.5 \mu \mathrm{m}$ & 1.00 & 0.380 & 0.940 \\
\hline $5.8 \mu \mathrm{m}$ & 1.49 & 0.207 & 0.720 \\
\hline $8.0 \mu \mathrm{m}$ & 1.37 & 0.330 & 0.740 \\
\hline
\end{tabular}

NOTE.-See $\S 3$ and http://spider.ipac.caltech.edu/staff/jarrett/irac/.

been respectively boosted by factors of $1.018,1.107$, and 1.049 , and their systematic uncertainties have dropped to $4 \%, 7 \%$, and 12\% (C. W. Engelbracht et al. 2007, in preparation; K. D. Gordon et al. 2007, in preparation; J. Stansberry et al. 2007, in preparation). The uncertainties provided in Table 3 include both calibration and statistical uncertainties. Calibration uncertainties are 5\%-10\% for IRAC 3.6 and $4.5 \mu \mathrm{m}$ data and $10 \%-15 \%$ for IRAC 5.8 and $8.0 \mu \mathrm{m}$ data; $10 \%$ calibration uncertainties are used in Table 3 .

The IRAC flux densities in Table 3 include extended source aperture corrections provided by the Spitzer Science Center. ${ }^{19}$ These corrections account for the "extended" emission due to the wings of the point-spread function (PSF) and also for the scattering of the diffuse emission across the IRAC focal plane. For an effective aperture radius $r=(a b)^{1 / 2}$ in arcseconds derived from the semimajor $a$ and semiminor $b$ ellipse axes provided in Table 1 , the IRAC extended source aperture correction is

$$
f_{\text {true }}^{\mathrm{IRAC}} / f_{\text {measured }}^{\mathrm{IRAC}}=A e^{-r^{B}}+C,
$$

where $A, B$, and $C$ are listed in Table 4 . The average extended source aperture corrections ( $\sim 10 \%$ uncertain) for the SINGS IRAC photometry are $[0.912,0.942,0.805,0.749]$ at $[3.6,4.5$, $5.8,8.0] \mu \mathrm{m}$.

The MIPS flux densities in Table 3 also include extended source aperture corrections. Three high-resolution models of a galaxy's structure are generated by convolving an $R$-band image of the galaxy with the MIPS PSFs. The MIPS aperture corrections listed in Table 5 are computed for the same apertures used in the global flux extractions (Table 1). The median aperture corrections are $[1.01,1.04,1.10]$ at $[24,70,160] \mu \mathrm{m}$. The uncertainties in the aperture corrections are typically a few percent and are based on the differences between the canonical and "minimum" corrections. Minimum MIPS aperture corrections are computed assuming point-source light distributions.

Finally, a correction for $70 \mu \mathrm{m}$ nonlinearity effects is included in this presentation. A preliminary correction of the form

$$
f_{\text {true }}^{70 \mu \mathrm{m}}=0.581\left(f_{\text {measured }}^{70 \mu \mathrm{m}}\right)^{1.13}
$$

derived from data presented by K. D. Gordon et al. (2007, in preparation), is applied to pixel values above a threshold of $\sim 66 \mathrm{MJy} \mathrm{sr}^{-1}$. A small fraction of the pixels in a total of 40 SINGS $70 \mu \mathrm{m}$ images require such a correction. The median correction to the global $70 \mu \mathrm{m}$ flux density for these 40 galaxies is a factor of 1.03, with the three largest corrections being factors of 1.124 (NGC 4826), 1.128 (NGC 1482), and 1.158 (NGC 7552).

\subsection{Radio Data}

Global $20 \mathrm{~cm}$ continuum fluxes from the literature are available for 62 SINGS galaxies, with data for 51 of these galaxies

\footnotetext{
19 See http://spider.ipac.caltech.edu/staff/jarrett/irac/.
}

TABLE 5

\begin{tabular}{|c|c|c|c|c|c|}
\hline Galaxy & $24 \mu \mathrm{m}$ & $70 \mu \mathrm{m}$ & $160 \mu \mathrm{m}$ & $450 \mu \mathrm{m}$ & $850 \mu \mathrm{m}$ \\
\hline 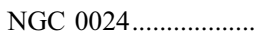 & 1.06 & 1.10 & 1.20 & $\ldots$ & $\ldots$ \\
\hline 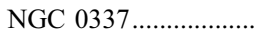 & 1.01 & 1.06 & 1.15 & $\ldots$ & $\ldots$ \\
\hline 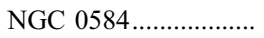 & 1.00 & 1.04 & 1.11 & $\ldots$ & $\ldots$ \\
\hline NGC $0628 \ldots \ldots \ldots \ldots \ldots . . . . . . .$. & 1.02 & 1.03 & 1.06 & $\ldots$ & $\ldots$ \\
\hline NGC $0855 \ldots \ldots \ldots \ldots . . . . . . . .$. & 1.02 & 1.07 & 1.15 & $\ldots$ & $\ldots$ \\
\hline 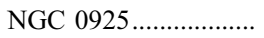 & 1.03 & 1.04 & 1.07 & $\ldots$ & $\ldots$ \\
\hline 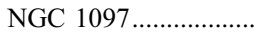 & 1.01 & 1.02 & 1.06 & $\ldots$ & 2.09 \\
\hline NGC $1266 \ldots \ldots \ldots \ldots . . . . . . . . .$. & 1.01 & 1.05 & 1.13 & $\ldots$ & $\ldots$ \\
\hline 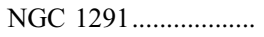 & 1.01 & 1.02 & 1.04 & $\ldots$ & $\ldots$ \\
\hline NGC $1316 \ldots \ldots \ldots \ldots . . . . . . . .$. & 1.06 & 1.02 & 1.18 & $\ldots$ & $\ldots$ \\
\hline 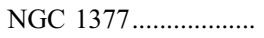 & 1.02 & 1.06 & 1.15 & $\ldots$ & $\ldots$ \\
\hline 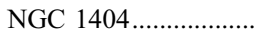 & 1.00 & 1.02 & 1.07 & $\ldots$ & $\ldots$ \\
\hline NGC $1482 \ldots \ldots \ldots \ldots \ldots . . . . . . .$. & 1.00 & 1.03 & 1.10 & $\ldots$ & $\ldots$ \\
\hline 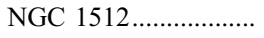 & 1.04 & 1.05 & 1.06 & $\ldots$ & $\ldots$ \\
\hline 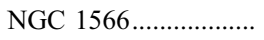 & 1.04 & 1.05 & 1.04 & $\ldots$ & $\ldots$ \\
\hline NGC $1705 \ldots \ldots \ldots \ldots \ldots . . . . . . . .$. & 1.04 & 1.12 & 1.19 & $\ldots$ & $\ldots$ \\
\hline 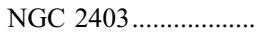 & 1.00 & 1.00 & 1.02 & $\ldots$ & $\ldots$ \\
\hline Holmberg II................ & 1.00 & 1.02 & 1.09 & $\ldots$ & $\ldots$ \\
\hline M81 Dwarf A ............. & 1.00 & 1.00 & 1.00 & $\ldots$ & $\ldots$ \\
\hline 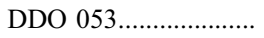 & 1.03 & 1.15 & 1.48 & $\ldots$ & $\ldots$ \\
\hline 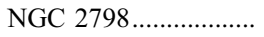 & 1.03 & 1.08 & 1.19 & $\ldots$ & 1.08 \\
\hline NGC $2841 \ldots \ldots \ldots \ldots \ldots . . . . . . . .$. & 1.01 & 1.04 & 1.10 & $\ldots$ & $\ldots$ \\
\hline 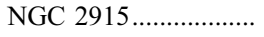 & 1.07 & 1.15 & 1.33 & $\ldots$ & $\ldots$ \\
\hline Holmberg I.................. & 1.01 & 1.05 & 1.15 & $\ldots$ & $\ldots$ \\
\hline 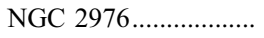 & 1.00 & 1.03 & 1.10 & $\ldots$ & 1.56 \\
\hline NGC $3049 \ldots \ldots \ldots \ldots . . . . . . . .$. & 1.02 & 1.07 & 1.18 & $\ldots$ & $\ldots$ \\
\hline NGC $3031 \ldots \ldots \ldots \ldots . . . . . . . . .$. & 1.00 & 1.00 & 1.01 & $\ldots$ & $\ldots$ \\
\hline 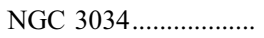 & 1.00 & 1.00 & 1.00 & $\ldots$ & $\ldots$ \\
\hline Holmberg IX ............... & 1.00 & 1.00 & 1.00 & $\ldots$ & $\ldots$ \\
\hline M81 Dwarf B.............. & 1.12 & 1.16 & 1.86 & $\ldots$ & $\ldots$ \\
\hline 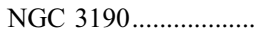 & 1.01 & 1.05 & 1.13 & $\ldots$ & 1.12 \\
\hline 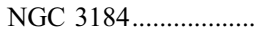 & 1.00 & 1.00 & 1.05 & $\ldots$ & $\ldots$ \\
\hline 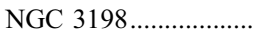 & 1.00 & 1.02 & 1.08 & $\ldots$ & $\ldots$ \\
\hline 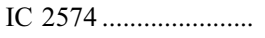 & 1.04 & 1.07 & 1.12 & $\ldots$ & $\ldots$ \\
\hline 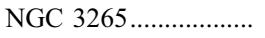 & 1.02 & 1.07 & 1.16 & $\ldots$ & $\ldots$ \\
\hline Mrk 33 ........................ & 1.02 & 1.07 & 1.16 & $\ldots$ & $\ldots$ \\
\hline 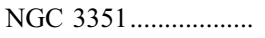 & 1.04 & 1.06 & 1.11 & $\ldots$ & $\ldots$ \\
\hline 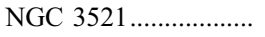 & 1.00 & 1.01 & 1.05 & $\ldots$ & 1.56 \\
\hline NGC $3621 \ldots \ldots \ldots \ldots . . . . . . . . .$. & 1.06 & 1.07 & 1.09 & $\ldots$ & $\ldots$ \\
\hline 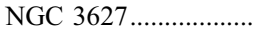 & 1.00 & 1.01 & 1.06 & $\ldots$ & 1.53 \\
\hline 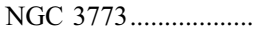 & 1.07 & 1.13 & 1.15 & $\ldots$ & $\ldots$ \\
\hline 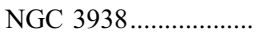 & 1.01 & 1.03 & 1.08 & $\ldots$ & $\ldots$ \\
\hline 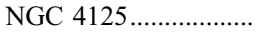 & 1.09 & 1.16 & 1.31 & $\ldots$ & $\ldots$ \\
\hline 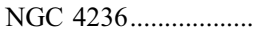 & 1.00 & 1.02 & 1.06 & $\ldots$ & $\ldots$ \\
\hline 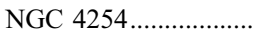 & 1.00 & 1.02 & 1.07 & $\ldots$ & 2.06 \\
\hline 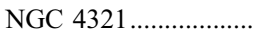 & 1.00 & 1.01 & 1.06 & $\ldots$ & 2.19 \\
\hline 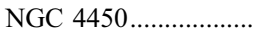 & 1.04 & 1.08 & 1.16 & $\ldots$ & $\ldots$ \\
\hline 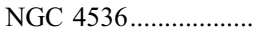 & 1.00 & 1.02 & 1.08 & $\ldots$ & 1.30 \\
\hline NGC $4552 \ldots \ldots \ldots \ldots \ldots \ldots . . . . . . .$. & 1.00 & 1.03 & 1.11 & $\cdots$ & $\ldots$ \\
\hline 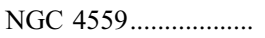 & 1.00 & 1.03 & 1.09 & $\ldots$ & $\ldots$ \\
\hline 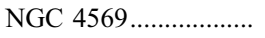 & 1.00 & 1.01 & 1.07 & $\ldots$ & 1.11 \\
\hline 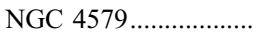 & 1.01 & 1.04 & 1.07 & $\ldots$ & $\ldots$ \\
\hline 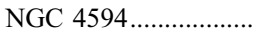 & 1.06 & 1.09 & 1.17 & $\ldots$ & 1.33 \\
\hline 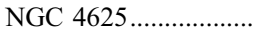 & 1.01 & 1.06 & 1.16 & $\ldots$ & $\ldots$ \\
\hline 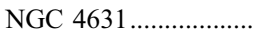 & 1.00 & 1.00 & 1.05 & 1.27 & 1.17 \\
\hline 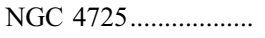 & 1.03 & 1.05 & 1.10 & $\ldots$ & $\ldots$ \\
\hline 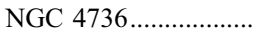 & 1.00 & 1.00 & 1.02 & $\ldots$ & 1.67 \\
\hline 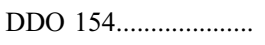 & 1.06 & 1.14 & 1.35 & $\cdots$ & $\ldots$ \\
\hline 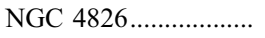 & 1.08 & 1.09 & 1.14 & $\ldots$ & 1.24 \\
\hline 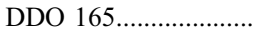 & 1.02 & 1.09 & 1.24 & $\ldots$ & $\ldots$ \\
\hline 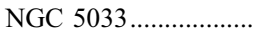 & 1.00 & 1.01 & 1.05 & $\ldots$ & 1.93 \\
\hline NGC $5055 \ldots \ldots \ldots \ldots \ldots \ldots . . . . . . . .$. & 1.00 & 1.00 & 1.03 & $\ldots$ & $\ldots$ \\
\hline 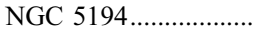 & 1.00 & 1.00 & 1.01 & $\ldots$ & $\ldots$ \\
\hline NGC $5195 \ldots \ldots \ldots \ldots \ldots . . . . . . . . .$. & 1.01 & 1.06 & 1.13 & $\ldots$ & $\ldots$ \\
\hline 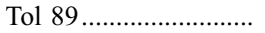 & 1.08 & 1.14 & 1.22 & $\ldots$ & $\ldots$ \\
\hline
\end{tabular}

Infrared and Submillimeter Aperture Correction Factors 
TABLE 5-Continued

\begin{tabular}{|c|c|c|c|c|c|}
\hline Galaxy & $24 \mu \mathrm{m}$ & $70 \mu \mathrm{m}$ & $160 \mu \mathrm{m}$ & $450 \mu \mathrm{m}$ & $850 \mu \mathrm{m}$ \\
\hline NGC $5408 \ldots \ldots \ldots \ldots \ldots \ldots$ & 1.01 & 1.05 & 1.12 & $\ldots$ & . \\
\hline 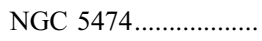 & 1.00 & 1.03 & 1.09 & $\ldots$ & $\ldots$ \\
\hline 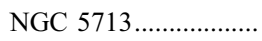 & 1.01 & 1.06 & 1.14 & $\ldots$ & 1.17 \\
\hline 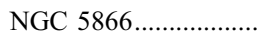 & 1.00 & 1.03 & 1.09 & $\ldots$ & $\ldots$ \\
\hline IC $4710 \ldots \ldots \ldots \ldots$ & 1.03 & 1.08 & 1.19 & $\ldots$ & $\ldots$ \\
\hline 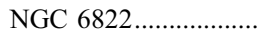 & 1.00 & 1.00 & 1.01 & $\ldots$ & $\ldots$ \\
\hline NGC 6946.................... & 1.00 & 1.00 & 1.03 & $\ldots$ & $\ldots$ \\
\hline NGC $7331 \ldots \ldots \ldots \ldots$ & 1.08 & 1.10 & 1.16 & 1.44 & 1.11 \\
\hline NGC $7552 \ldots \ldots \ldots \ldots \ldots . . .$. & 1.01 & 1.02 & 1.13 & $\ldots$ & 1.17 \\
\hline NGC $7793 \ldots \ldots \ldots \ldots \ldots . . .$. & 1.01 & 1.03 & 1.08 & $\ldots$ & . \\
\hline
\end{tabular}

Notes.-IRAC aperture corrections are described by eq. (2). See $\S 3.3$ and Dale et al. (2005) for details.

taken from the New VLA Sky Survey catalog (Condon et al. 1998; Yun et al. 2001; see Table 3). Although this is a snapshot survey and prone to miss extended emission from galaxies having large angular extents, proper attention has been paid to these effects to derive unbiased 1.4 GHz fluxes (e.g., Yun et al. 2001). The $20 \mathrm{~cm}$ data for 11 additional galaxies were taken from Condon
(1987), Hummel (1980), Condon et al. (1990), Wright \& Otrupcek (1990), Bauer et al. (2000), and Cannon et al. (2006b).

\section{RESULTS}

\subsection{Global Broadband Spectral Energy Distributions}

Figure 1 shows the ultraviolet-to-submillimeter SEDs for the SINGS sample. The solid line is the sum of a dust (dashed line) and a stellar (dotted line) model. The dust curve is a Dale \& Helou (2002) model (least squares) fitted to ratios of the 24, 70, and $160 \mu \mathrm{m}$ fluxes (a dust curve for NGC 3034 is fitted using IRAS 25,60 , and $100 \mu \mathrm{m}$ data, since the MIPS data for this galaxy are saturated). The $\alpha_{\text {SED }}$ listed within each panel parameterizes the distribution of dust mass as a function of heating intensity, as described in Dale \& Helou (2002). Broadly speaking, smaller values of $\alpha_{\text {SED }}$ (more heating from stronger interstellar radiation fields) correspond to later Hubble types and larger infrared-toultraviolet ratios. The stellar curve is a 1 Gyr continuous star formation, solar metallicity curve from Vazquez \& Leitherer (2005) fitted to the 2MASS data. The initial mass function for this curve utilizes a double power-law form, with $\alpha_{1, \mathrm{IMF}}=1.3$ for $0.1<$ $m / M_{\odot}<0.5$ and $\alpha_{2, \mathrm{IMF}}=2.3$ for $0.5<m / M_{\odot}<100$ (e.g.,

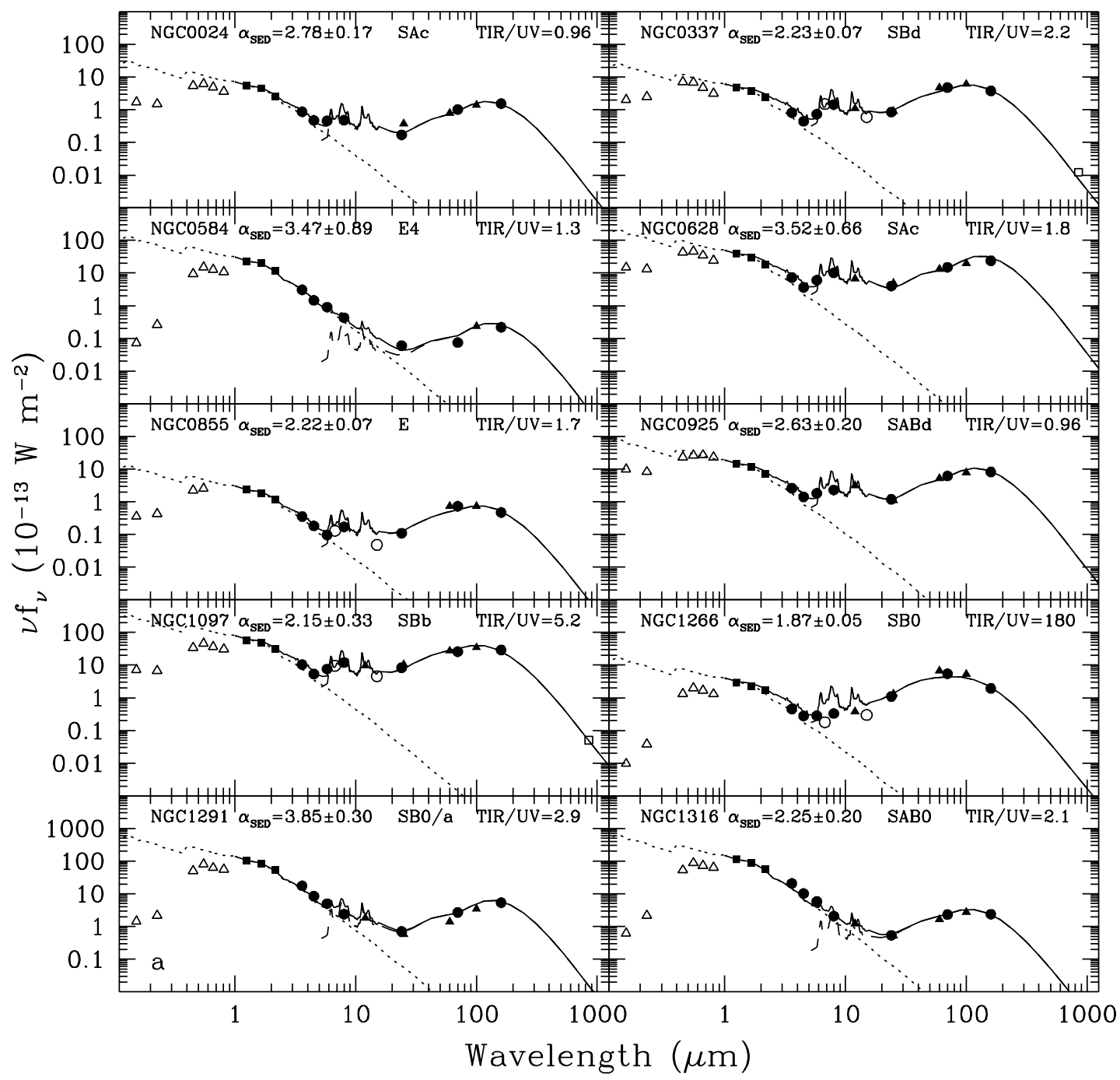

FIG. 1.-Globally integrated $0.15-850 \mu \mathrm{m}$ SEDs for the SINGS sample. GALEX and optical, 2MASS, Spitzer, IRAS, ISO, and SCUBA data are represented by open triangles, filled squares, filled circles, filled triangles, open circles, and open squares, respectively. The solid line is the sum of a dust (dashed line) and a stellar (dotted line) model. The dust curve is a Dale \& Helou (2002) model fitted to ratios of the 24, 70, and $160 \mu$ m fluxes; the $\alpha_{\mathrm{SED}}$ listed within each panel parameterizes the distribution of dust mass as a function of heating intensity, as described in Dale \& Helou (2002). The stellar curve is a 1 Gyr continuous star formation, solar metallicity curve from Vazquez \& Leitherer (2005) fitted to the 2MASS data (see $\S 4$ for details). [See the electronic edition of the Journal for a color version of this figure.] 


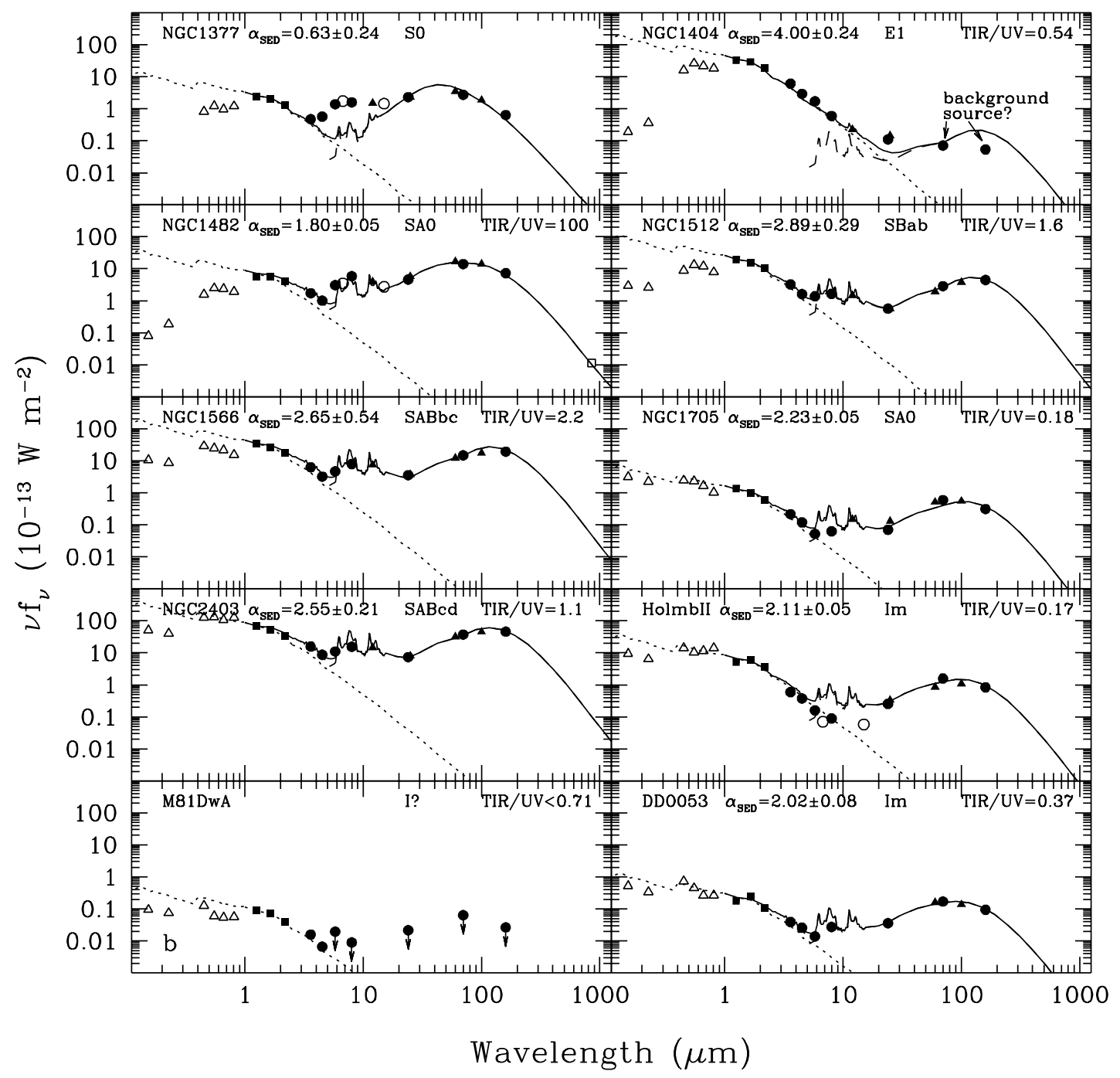

FIG. 1.-Continued

Kroupa 2002). Although this stellar curve (not adjusted for internal extinction) may not be applicable to many galaxies, especially elliptical galaxies, it is included as a "standard" reference against which deviations in the ultraviolet and optical can be compared from galaxy to galaxy. The stellar curve also serves to highlight the relative importance of stars and dust in each galaxy, particularly in the transition from stellar to dust emission in the mid-infrared (e.g., NGC 1404 vs. NGC 1482).

Several galaxies show mid-infrared data that deviate from the fits. Most of these systems are low-metallicity objects (e.g., Holmberg II, NGC 2915, IC 2574, DDO 154, DDO 165, and NGC 6822), objects that have been shown to be deficient in polycyclic aromatic hydrocarbon (PAH) emission (see the discussion in $\S$ 4.2). The mid-infrared data for NGC 1377 are also quite discrepant from the model, showing a strong excess for each of the broadband filters from 3.6 to $15 \mu \mathrm{m}$. The substantial hot dust emission and lack of optical signatures or synchrotron radiation led Roussel et al. (2003) to infer that this heavily extincted system is undergoing the very beginnings of an intense burst of star formation.

\subsection{Spectral Energy Distributions Binned by the Infrared-to-Ultraviolet Ratio}

Analysis of the distribution of global (spatially integrated) SEDs is a sensible starting point for current cosmology surveys (e.g., Rowan-Robinson et al. 2005). Figure 2 shows a stack of SINGS SEDs that emphasizes the infrared-to-ultraviolet variations within the SINGS sample. Each SED in the stack represents an average of approximately 10 individual SEDs that fall within a given bin of the total infrared-to-ultraviolet ratio [TIR/(FUV+ NUV); the bins are indicated in the figure legend]. The ultraviolet emission for this ratio is computed as $\nu f_{\nu}(1500 \AA)+$ $\nu f_{\nu}(2300 \AA)$, whereas the "total infrared" is the dust continuum emission between 3 and $1100 \mu \mathrm{m}$ (Dale et al. 2001), computed using the MIPS 24, 70, and $160 \mu \mathrm{m}$ fluxes and equation (4) of Dale \& Helou (2002). The spectra are arbitrarily normalized at the 2MASS $K_{s}$ band wavelength.

Several features in the stack are immediately noticeable. The ultraviolet slopes vary from positive values for galaxies with high infrared-to-ultraviolet ratios to negative values for low infraredto-ultraviolet ratio galaxies (as explored in detail in $\S 5.6$ ). The $4000 \AA$ break shows up quite clearly, even at this coarse spectral "resolution." Other obvious features include the broad far-infrared peak, signifying emission from cool to warm large grains; the contributions from PAHs appearing as mid-infrared emission features; the near-infrared hump arising from photospheric emission from old stellar populations; and a near-infrared $\mathrm{H}$ - opacity signature for high infrared-to-ultraviolet systems. Note also the broad spread in the ultraviolet data compared to that in the far-infrared. Since the 


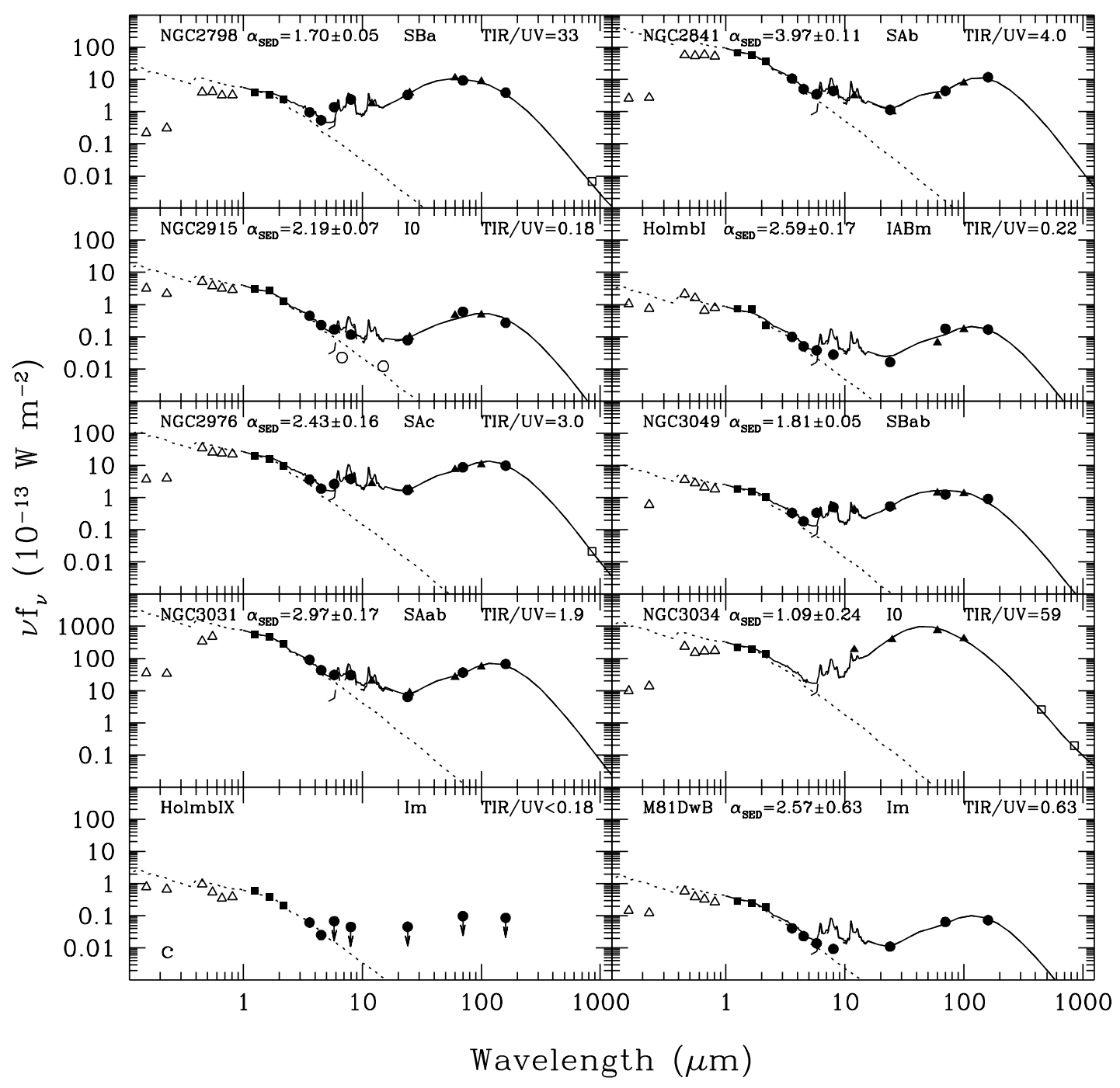

FIG. 1.-Continued

spectra are normalized at $K_{s}$, this difference implies that there is a larger spread in ultraviolet light per unit stellar mass than infrared light per unit stellar mass (Gil de Paz et al. [2007], for example, find that $[\mathrm{FUV}-K]$ spans $11 \mathrm{mag}$ in their $G A L E X$ Atlas ). The variations in the infrared-to-ultraviolet ratio studied later in this work are largely driven by variations in the ultraviolet emission.

Close inspection of Figure 2 reveals that most of the variation in the stacked spectra stems from the two extreme bins (bins " 1 " and " 6 ") in the infrared-to-ultraviolet ratio. However, substantial variations are still seen in bins $2-5$ at ultraviolet and mid-infrared wavelengths. The bin $2-5$ range is $0.88,0.78,0.24$, and 0.16 dex at $0.15,0.23,8.0$, and $24 \mu \mathrm{m}$, respectively (compared to the bin 1-6 range of $1.76,1.46,0.80$, and $0.80 \mathrm{dex}$ at the same wavelengths). The spread at ultraviolet wavelengths is presumably significantly affected by variations in dust extinction. The range in $8.0 \mu \mathrm{m}$ emission, on the other hand, is likely due to PAH destruction/ formation variations. Low-metallicity systems, for example, are known to be deficient in PAH emission (e.g., Dale et al. 2005; Engelbracht et al. 2005; Galliano et al. 2005; Walter et al. 2006). Indeed, eight of the nine galaxies in the lowest infrared-toultraviolet ratio bin have low metallicities $[12+\log (\mathrm{O} / \mathrm{H})<$ 8.1; J. Moustakas et al. 2007, in preparation], and this bin's average spectrum in Figure 2 shows very low mid-infrared emission. The $24 \mu \mathrm{m}$ emission from galaxies is known to be sensitive to the star formation rate (e.g., Dale et al. 2005; Gordon et al. 2004; Helou et al. 2004; Hinz et al. 2004; Calzetti et al. 2005); the observed variations at this wavelength may be strongly affected by the range in the sample's star formation properties.

\subsection{Principal Component Analysis}

A principal component analysis can help to quantify relative contributions to the observed variations in a sample of SEDs (Deeming 1964). A set of $i$ eigenvectors $\left\{\boldsymbol{e}_{i}\right\}$ and their corresponding eigenvalues $\left\{e_{i}\right\}$ for our sample of $N$ galaxies are computed from a diagonalization of the covariance matrix

$$
C_{j k}=\frac{1}{N} \Sigma_{i=1}^{N} \nu f_{\nu}^{i}\left(\lambda_{j}\right) \nu f_{\nu}^{i}\left(\lambda_{k}\right)
$$

where $\nu f_{\nu}^{i}\left(\lambda_{j}\right)$ is the flux of the $i$ th spectrum at wavelength $\lambda_{j}$. We restrict the computation of the covariance matrix to involve only those wavelengths for which we have a substantial database of fluxes; submillimeter data at 450 and $850 \mu \mathrm{m}$ are not included in the principal component analysis. Furthermore, to avoid spurious results, we do not include in our analysis any SINGS galaxies without a secure detection/measurement at any of the ultraviolet, optical, near-infrared, or infrared wavelengths listed in 


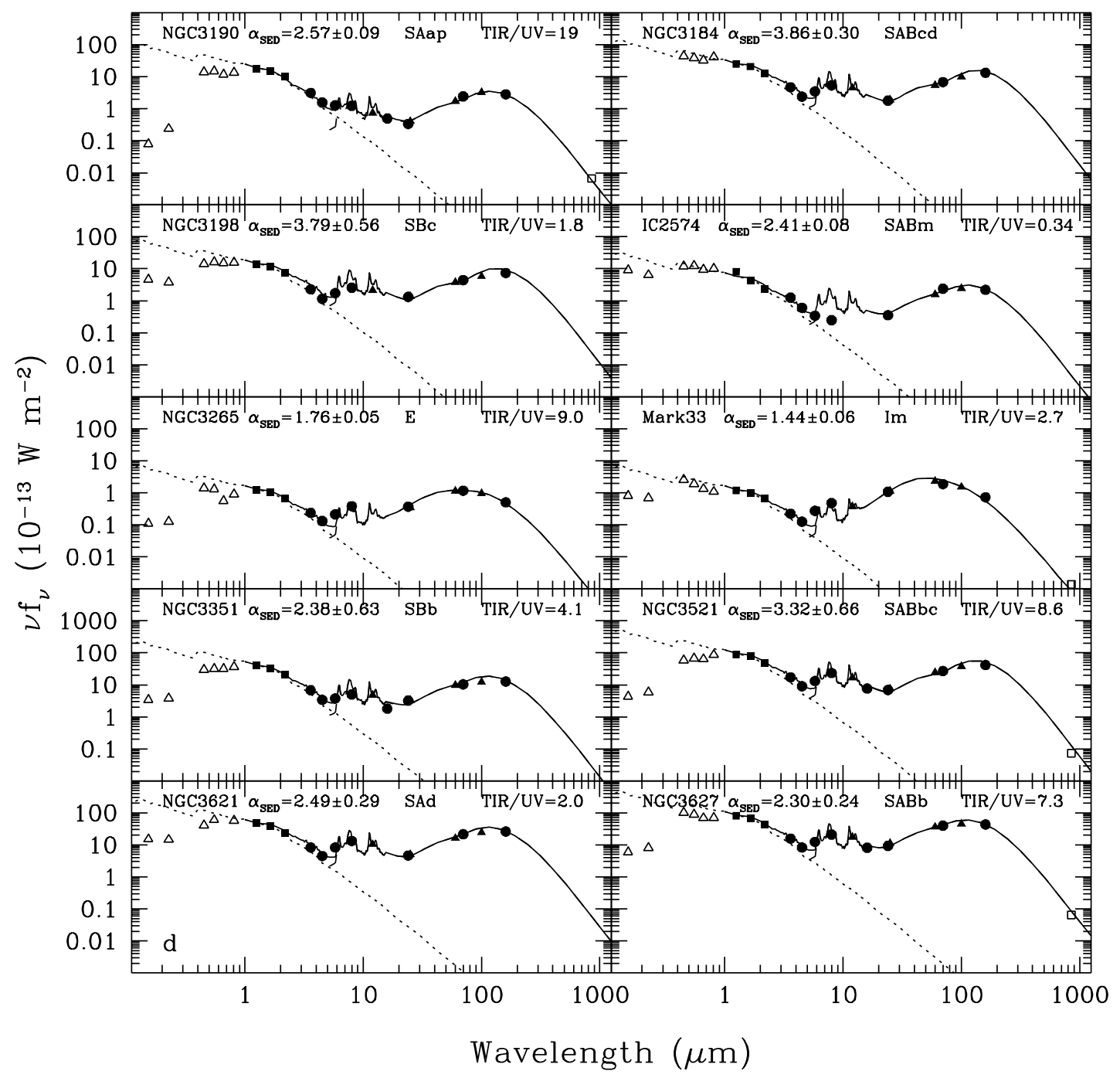

FIG. 1.-Continued

Tables 2 and 3. Hence, our principal component analysis involves only about three-fourths of the SINGS sample (Table 1 indicates which systems are involved). Our principal component analysis is carried out after normalizing the spectra at the 2MASS $K_{s}$ band wavelength.

The principal component analysis has produced eigenvectors that describe different components of the sample spectra (Fig. 3). The most important eigenvectors are the first two. Eigenvector $\boldsymbol{e}_{1}$ appears to describe the contribution of unobscured starlight (including starlight from star formation regions) to the galaxies' spectra. This eigenvector also includes additional low-level farinfrared emission that may represent cirrus emission from the diffuse interstellar medium. Eigenvector $\boldsymbol{e}_{2}$ appears to describe the effects of dust on the shape of the spectrum. This eigenvector demonstrates that as the dust content of the galaxies increases, the midand far-infrared flux densities increase while the starlight becomes redder (i.e., the ultraviolet and blue light decreases while the red and near-infrared light increases).

The individual eigenvalues normalized by the sum $\Sigma_{j} e_{j}$ of all eigenvalues indicate the fractional contribution of each corresponding eigenvector to the variation in the spectral atlas (when normalized at $K_{s}$ ). The normalized $e_{1}$ and $e_{2}$ eigenvalues (which correspond to the $\boldsymbol{e}_{1}$ and $\boldsymbol{e}_{2}$ eigenvectors) are 0.88 and 0.07 , respectively. In other words, $\boldsymbol{e}_{1}$ and $\boldsymbol{e}_{2}$ represent $88 \%$ and $7 \%$ of the total variation in the spectral atlas. The remaining $5 \%$ of the variation is represented by additional eigenvectors with corresponding normalized eigenvalues that are individually 0.02 or smaller. To quantify the uncertainty on these numbers, we have performed 10,000 Monte Carlo simulations of the principal component analysis. For each simulation we use the tabulated flux uncertainties to add a random (Gaussian deviate) flux offset to every galaxy's flux at each wavelength. The means of the two largest normalized eigenvalues from these simulations are $\left\langle e_{1}\right\rangle=0.88 \pm 0.01$ and $\left\langle e_{2}\right\rangle=0.07 \pm 0.01$, with the error bars reflecting the standard deviations in the simulations. The eigenspectra and error bars in Figure 3 reflect the mean values and standard deviations in the simulations.

\section{THE INFRARED-TO-ULTRAVIOLET RATIO}

The infrared-to-ultraviolet ratio is a rough measure of the amount of extinction at ultraviolet wavelengths. The infrared-to-ultraviolet ratio in galaxies is also sensitive to the metal content, luminosity, star formation history, and the relative distribution of interstellar grains with respect to their heating sources. What is the predominant driver of the variations in this ratio in galaxies? Which parameters can be used to most easily quantify these variations, with the aim of simplifying SED analysis? Various possibilities are presented and discussed below. 


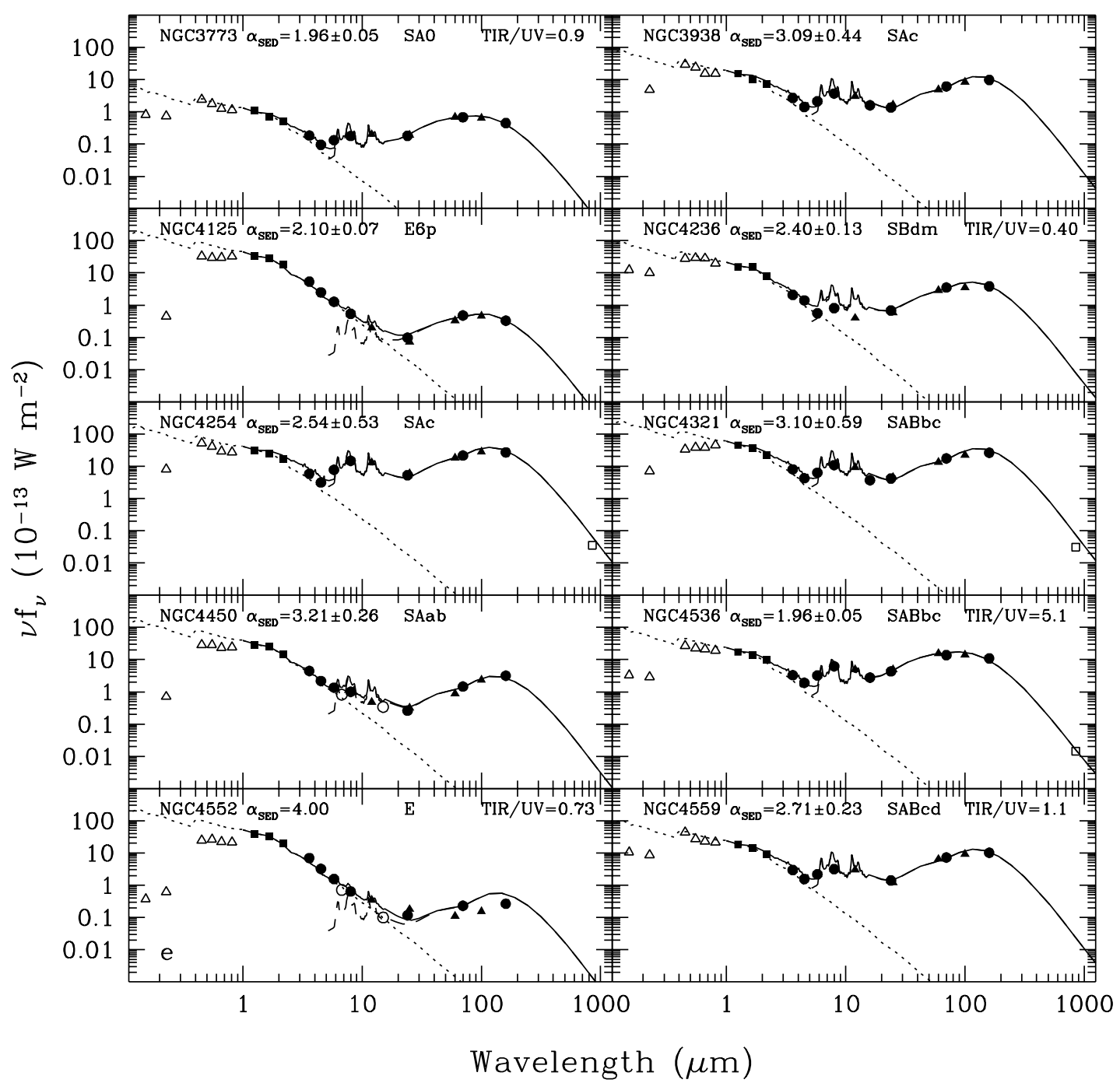

FIG. 1.-Continued

\subsection{Inclination}

The tilt of a spiral disk with respect to the observer's line of sight affects the observed intensity and colors (e.g., Bruzual et al. 1988; Boselli \& Gavazzi 1994; Giovanelli et al. 1995; Kuchinski et al. 1998). The "disk" inclination can be computed from the observed semimajor and semiminor axes, $a$ and $b$, assuming that disks are oblate spheroids with intrinsic axial ratio $(b / a)_{\text {int }}$ using the relation

$$
\cos ^{2} i=\frac{(b / a)^{2}-(b / a)_{\mathrm{int}}^{2}}{1-(b / a)_{\mathrm{int}}^{2}}
$$

where $(b / a)_{\text {int }} \simeq 0.2$ for morphological types earlier than Sbc and $(b / a)_{\text {int }} \simeq 0.13$ otherwise (see Dale et al. 1997 and references therein). Figure 4 gives the infrared-to-ultraviolet ratio as a function of galaxy disk inclination. Galaxies with elliptical and irregular morphologies have not been included in the plot. The dotted line (normalized to an infrared-to-ultraviolet ratio of unity at zero inclination) shows the expected effect of extinction on the ultraviolet data with changing inclination using the thin-disk model and a central face-on optical depth in the $B$ band of $\tau_{B}^{f}=2$ described in Tuffs et al. (2004). The ratio does not obviously trend with galaxy orientation; if there is a trend consistent with the model of Tuffs et al. (2004), it is a weak trend that is washed out by a large dispersion. The data in Figure 4 indicate that moderate disk inclinations are not a dominant factor in determining the infrared-to-ultraviolet ratio in SINGS galaxies.

\subsection{Hubble Type}

Figure 5 displays the infrared-to-ultraviolet ratio as a function of galaxy optical morphology. In general, the ultraviolet light increases in importance and the dust emission decreases in importance as the morphology changes from early-type spiral galaxies to late-type spiral galaxies to irregular galaxies, reflecting the changing significance of recent star formation and dust content/ distribution to the overall energy budget in galaxies (see also Buat \& Xu 1996; Gil de Paz et al. 2007). One interpretation is that the infrared-to-ultraviolet ratio increases as the redder, older stellar populations increasingly dominate for earlier type spiral galaxies, but as shown in $\S 5.3$, the increased porosity of star-forming regions for more actively star-forming galaxies may play a key role in this trend. Elliptical and S0 galaxies do not follow the general trend exhibited by the spiral and irregular galaxies; some elliptical and S0 galaxies show comparatively low infrared-toultraviolet ratios. This deviation to low infrared-to-ultraviolet ratios for some of the earliest type galaxies could be due to a relative paucity of dust grains intercepting ultraviolet/optical/near-infrared 


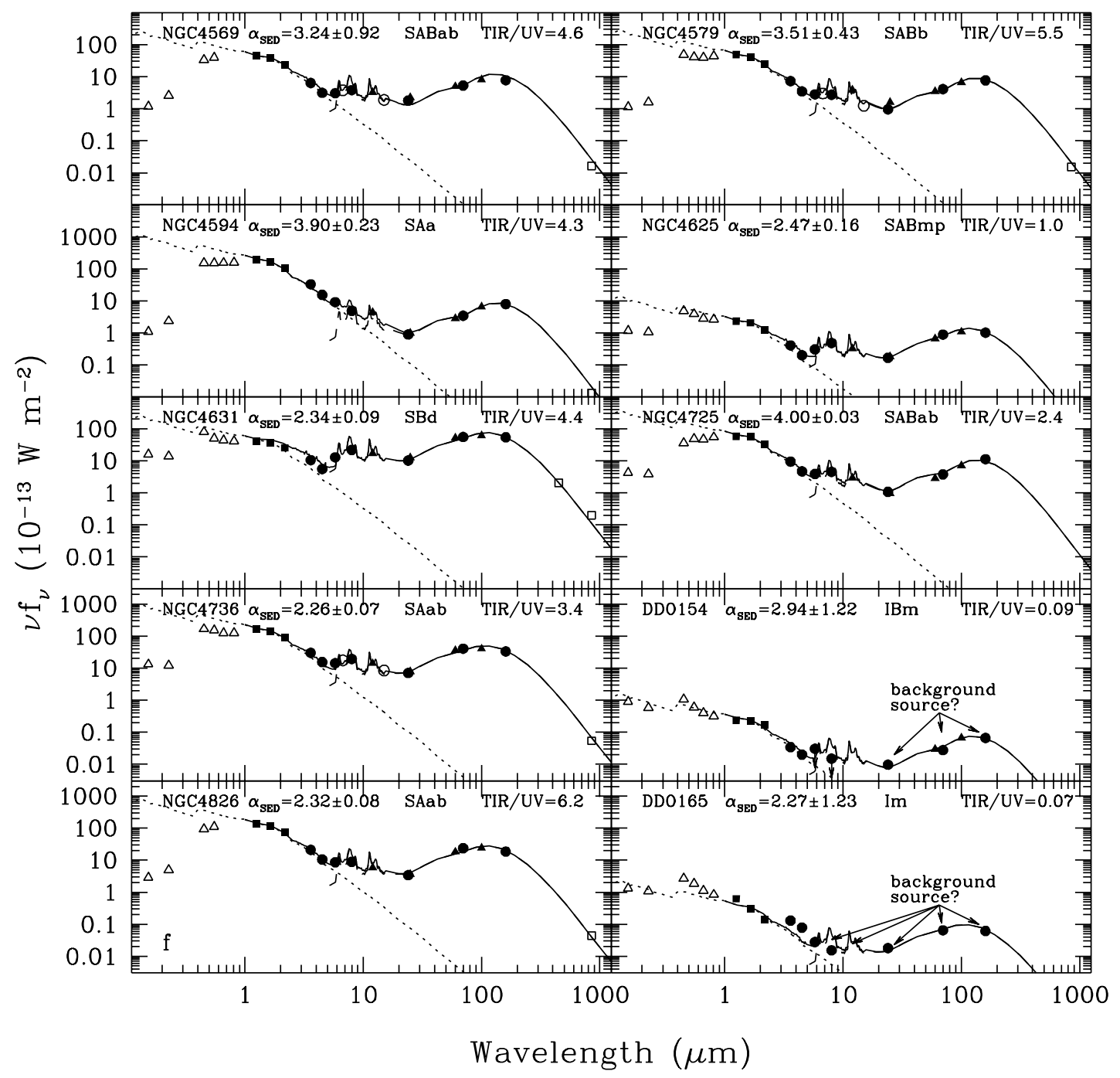

FIG. 1.-Continued

photons and reprocessing that energy into the infrared; the infrared portion of the bolometric luminosity in elliptical galaxies is typically only a few percent (Xilouris et al. 2004). Moreover, some elliptical systems are conspicuous ultraviolet emitters, with the emission thought to mainly arise from low-mass, heliumburning stars from the extreme horizontal branch and later phases of stellar evolution (for a review see O'Connell 1999). Low or moderate levels of star formation could also contribute to the ultraviolet emission in early-type galaxies (e.g., Fukugita et al. 2004). Recent evidence shows that strong ultraviolet emitters are the largest contributors to the significant scatter in the ultraviolet colors of early-type galaxies (e.g., Yi et al. 2005; Rich et al. 2005).

This wide range in the fractional ultraviolet luminosity also leads to significant scatter in the infrared-to-ultraviolet ratio. Although the statistics are based on small numbers, a similarly large dispersion is seen for irregular systems at the other end of the morphological spectrum. Part of this dispersion is likely associated with the metal content in irregular/dwarf systems. In general, irregular galaxies are quite blue and metal-poor (e.g., Hunter \& Gallagher 1986; van Zee et al. 1997). Ultraviolet/optical continuum emission from low-metallicity galaxies experiences less extinction, which inhibits the production of infrared continuum emission (see previous paragraph). The combination of these ef- fects leads to lower infrared-to-ultraviolet ratios in irregular galaxies. $^{20}$

\subsection{Far-Infrared Color}

Although dwarf irregular galaxies show low infrared-toultraviolet ratios, their interstellar dust grains tend to be vigorously heated. The lower metallicity in these systems results in less line blanketing, which in turn leads to harder radiation fields. Many of the dwarf and irregular systems in the SINGS sample indeed have elevated $f_{\nu}(70 \mu \mathrm{m}) / f_{\nu}(160 \mu \mathrm{m})$ ratios (e.g., Dale et al. 2005; Walter et al. 2006; see also Boselli et al. 2003), indicating strong overall heating of the dust grain population. The warmer far-infrared colors for SINGS dwarf/irregular galaxies are evident in Figure 6, which plots the infrared-to-ultraviolet ratio versus farinfrared color for the entire SINGS sample.

An interesting feature to this plot is the apparent wedge-shaped distribution, with a progressively smaller range in the infrared-toultraviolet ratio for cooler far-infrared colors. There is no obvious trend in infrared-to-ultraviolet ratio with disk inclination (Fig. 4), so it is unlikely that the distribution in Figure 6 is due solely to disk orientation.

\footnotetext{
20 The amorphous, starbursting M82 hosts a large infrared-to-ultraviolet ratio and should be considered separately from the dwarf irregular galaxies.
} 


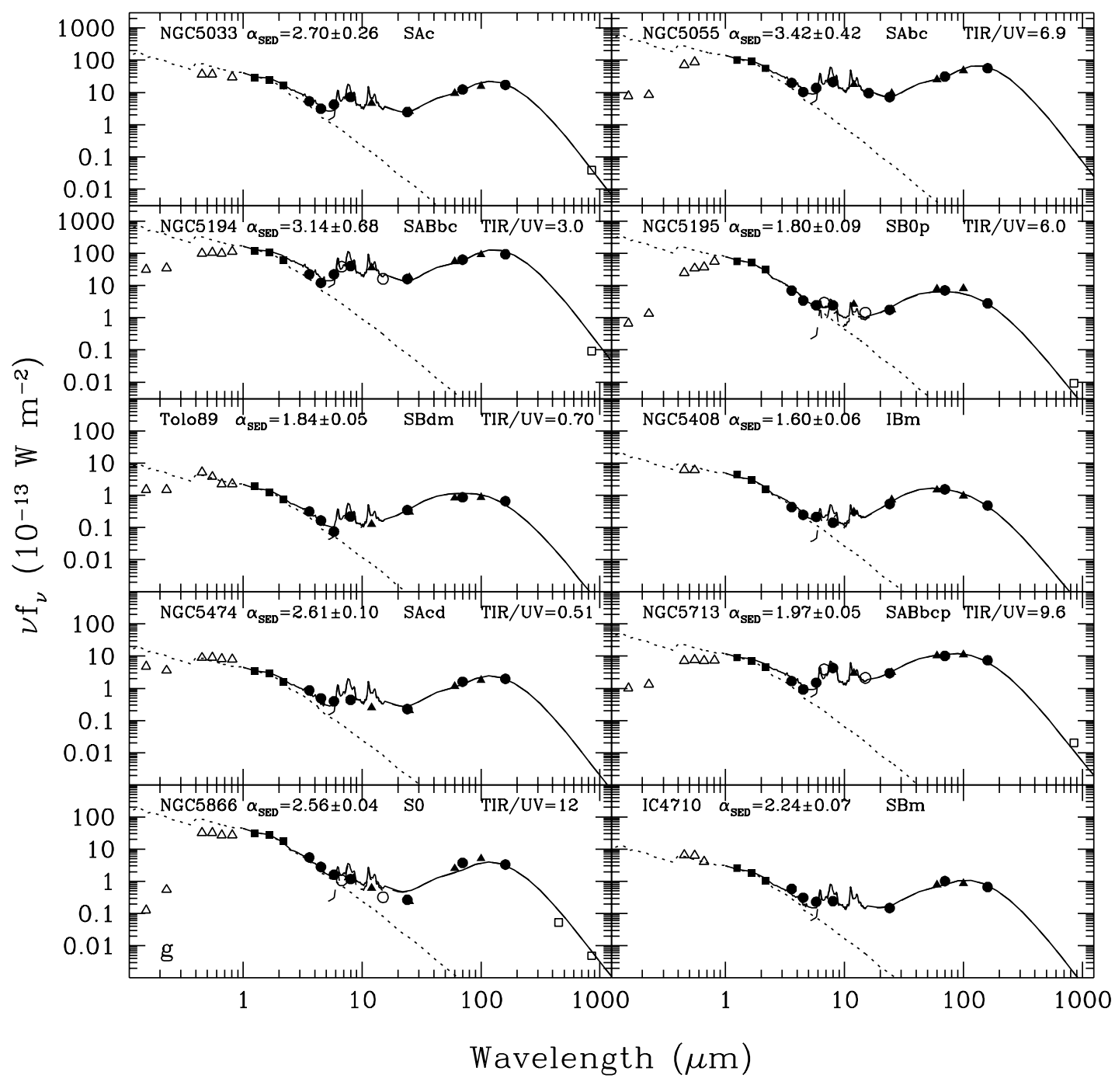

FIG. 1.-Continued

The small data points without error bars come from the (IRAS based) GALEX Atlas of Nearby Galaxies (Gil de Paz et al. 2007) and follow the same general distribution as the SINGS data, suggesting that this wedge-shaped distribution is unlikely a sample selection effect. Presumably the upper left portion of this figure, for example, is empty since a large infrared-to-ultraviolet ratio requires lots of dust opacity, but higher opacity implies a larger density of interstellar dust closer to heating sources, therefore leading to warm dust and high values of $f_{\nu}(70 \mu \mathrm{m}) / f_{\nu}(160 \mu \mathrm{m})$.

The relative distribution of dust grains and their heating sources may, in fact, play a key role in creating this overall wedge-shaped distribution. As argued above, it is reasonable to assume that galaxies with relatively high $f_{\nu}(70 \mu \mathrm{m}) / f_{\nu}(160 \mu \mathrm{m})$ ratios have hotter dust since the dust in such systems is near sites of active star formation or active nuclei. Moreover, galaxies that appear as several bright clumps in the infrared such as Holmberg II provide a large number of low optical depth lines of sight from which ultraviolet photons may escape (or their ultraviolet emission does not come from a single burst, but is rather more continuous or multigenerational in nature). Such clumpy galaxies would hence show comparatively low infrared-to-ultraviolet ratios. On the other hand, ultraviolet photons from galaxies that appear in the infrared as a single pointlike blob of nuclear emission (e.g., NGC 1266) would encounter significant extinction, and hence such galaxies would exhibit high infrared-to-ultraviolet ratios and high dust temperatures. In contrast to hot dust systems, galaxies with relatively low $f_{\nu}(70 \mu \mathrm{m}) / f_{\nu}(160 \mu \mathrm{m})$ ratios have cooler dust because the dust is not in spatial proximity of the hot stars (e.g., Panagia 1973). The heating of dust via the weaker ambient interstellar radiation field would be fractionally higher in these galaxies. Therefore, their morphological appearance in the infrared should be comparatively smooth (e.g., NGC 2841).

Since the relative distribution of interstellar grains and their heating sources is central to the scenario outlined above, we turn to the $24 \mu \mathrm{m}$ morphology of SINGS galaxies to provide a test of the above scenario. MIPS $24 \mu \mathrm{m}$ data may be uniquely suited for such a test, as the data have significantly higher spatial resolution than either 70 or $160 \mu \mathrm{m}$ imaging (the $6^{\prime \prime} 24 \mu \mathrm{m}$ beam corresponds to $\sim 0.3 \mathrm{kpc}$ at $10 \mathrm{Mpc}$ ) and effectively trace both interstellar grains and active sites of star formation ${ }^{21}$ (e.g., Hinz et al. 2004; Gordon et al. 2004). In fact, the $24 \mu \mathrm{m}$ emission can be spatially closely associated with $\mathrm{H}$ II regions and in such cases is probably dominated by dust from within these regions (Helou et al. 2004; Murphy et al. 2006). To facilitate our analysis, we have decomposed the $24 \mu \mathrm{m}$ images into unresolved (point sources) and

21 Note that the $24 \mu \mathrm{m}$ emission contains up to $\sim 25 \%$ stellar emission for many SINGS early-type galaxies. 


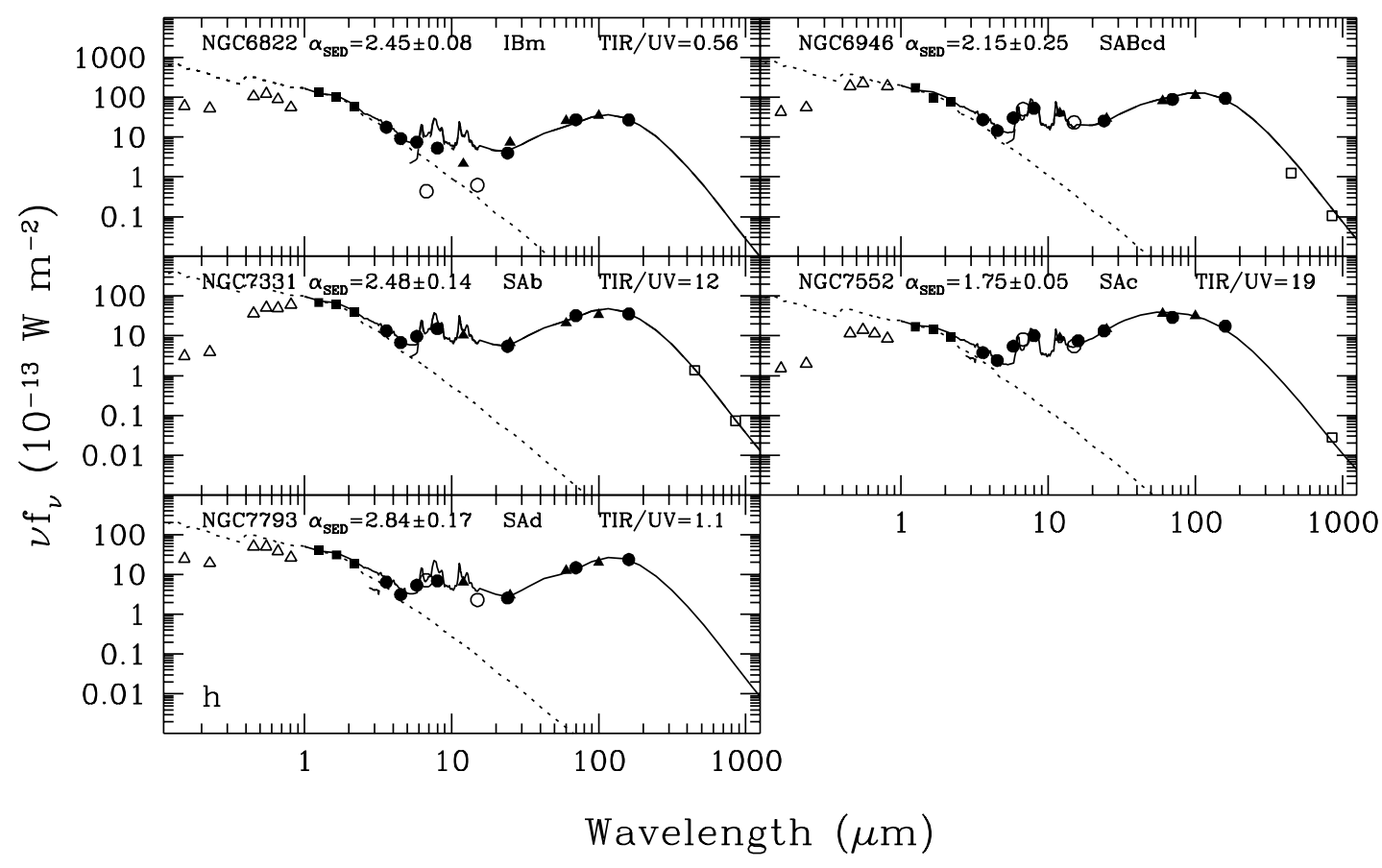

Fig. 1.-Continued

resolved emission. The point-source photometry is done using StarFinder (Diolaiti et al. 2000), which is appropriate for the stable and well-sampled MIPS $24 \mu \mathrm{m}$ PSF. A STinyTim (Krist 2002) model PSF with a temperature of $100 \mathrm{~K}$, smoothed to account for pixel sampling, is used. Smoothed STinyTim PSFs are excellent matches to observed MIPS $24 \mu \mathrm{m}$ PSFs (C. W. Engelbracht et al. 2007, in preparation). An image of all of the detected point sources is created along with a difference image made by subtracting the point-source image from the observed image. The fluxes are measured in the point-source ("unresolved") and difference ("resolved") images in the same aperture used for the total galaxy measurement (see Fig. 7). In addition, nuclear fluxes are measured in a $12^{\prime \prime}$ radius circular aperture on the observed image.

The results from this analysis are displayed in Figures 8 and 9. In Figure 8 the symbol size linearly scales with the ratio of unresolved to resolved $24 \mu \mathrm{m}$ emission, with the largest symbols corresponding to ratios $\sim 10$.

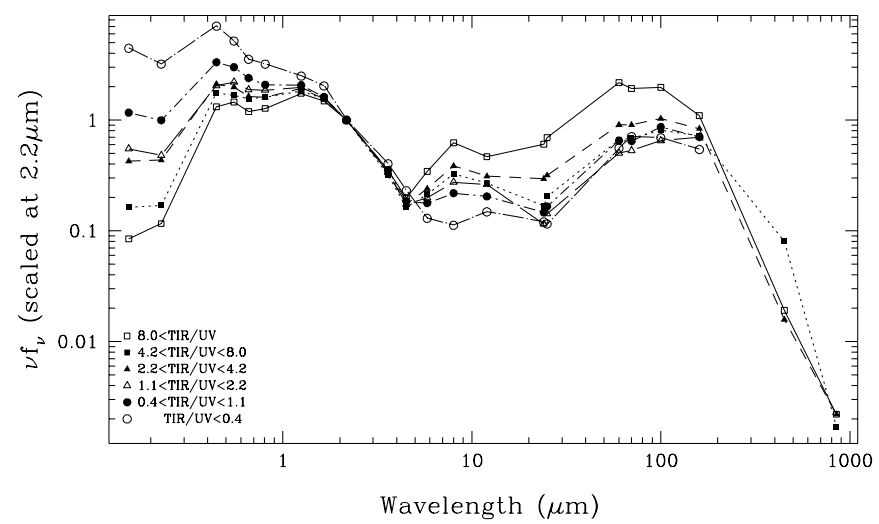

FIG. 2.-Display of stacked SEDs that emphasizes the infrared-to-ultraviolet variations within the SINGS sample. Each SED in the stack represents an average of approximately 10 individual SEDs that fall within a given bin of the infrared-to-ultraviolet ratio. [See the electronic edition of the Journal for a color version of this figure.]
In addition, each data symbol reflects the ratio of nuclear to total $24 \mu \mathrm{m}$ emission, as indicated in the figure legend. Galaxies dominated by a single point source of nuclear emission at $24 \mu \mathrm{m}$ appear preferentially in the upper right portion of the diagram. These galaxies contain hot dust and show relatively high infrared-to-ultraviolet ratios since the dust is centrally concentrated near the heating sources in the nuclei. Note that nuclear activity is not the main factor in determining the $24 \mu \mathrm{m}$ morphology: only two of the pointlike systems have active nuclei (NGC 1266 and NGC 5195). Systems with clumpy $24 \mu \mathrm{m}$ morphologies appearing in the lower right corner still contain hot dust; the dust is concentrated around several heating sources, not just the nuclear ones. Moreover, the clumpy distribution provides a larger number of low $\tau$ or "clean" lines of sight for ultraviolet photons to escape the

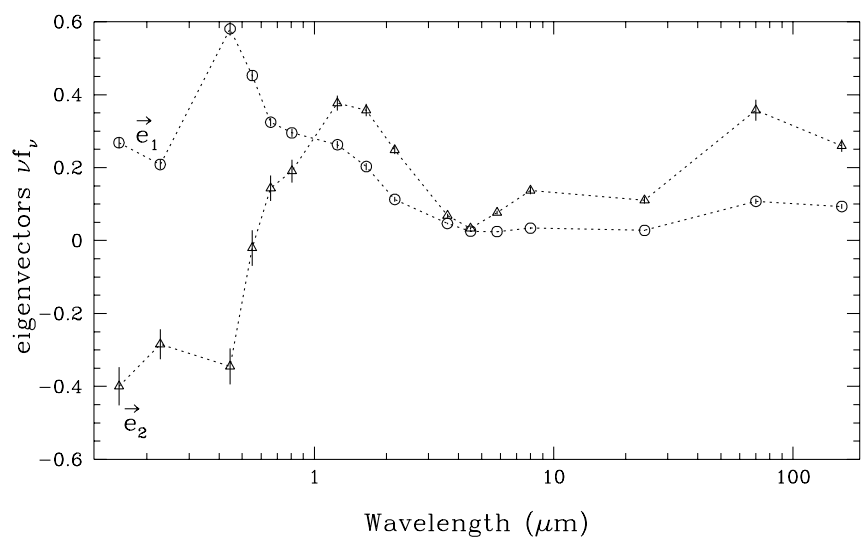

FIG. 3.-Strongest (circles) and second strongest (triangles) eigenvector spectra from a principal component analysis of the SINGS spectra. These are average eigenvectors stemming from 10,000 Monte Carlo simulations based on the observed fluxes and their uncertainties (corrected for Galactic extinction and air mass in the case of ground-based observations); the error bars shown in this figure indicate the dispersion of the eigenspectra from the simulations. These eigenvectors have normalized eigenvalues of 0.88 and $0.07 ;\left\langle\boldsymbol{e}_{1}\right\rangle$ and $\left\langle\boldsymbol{e}_{2}\right\rangle$, respectively, contribute to $88 \%$ and $7 \%$ of the observed variation in the sample spectra. [See the electronic edition of the Journal for a color version of this figure.] 


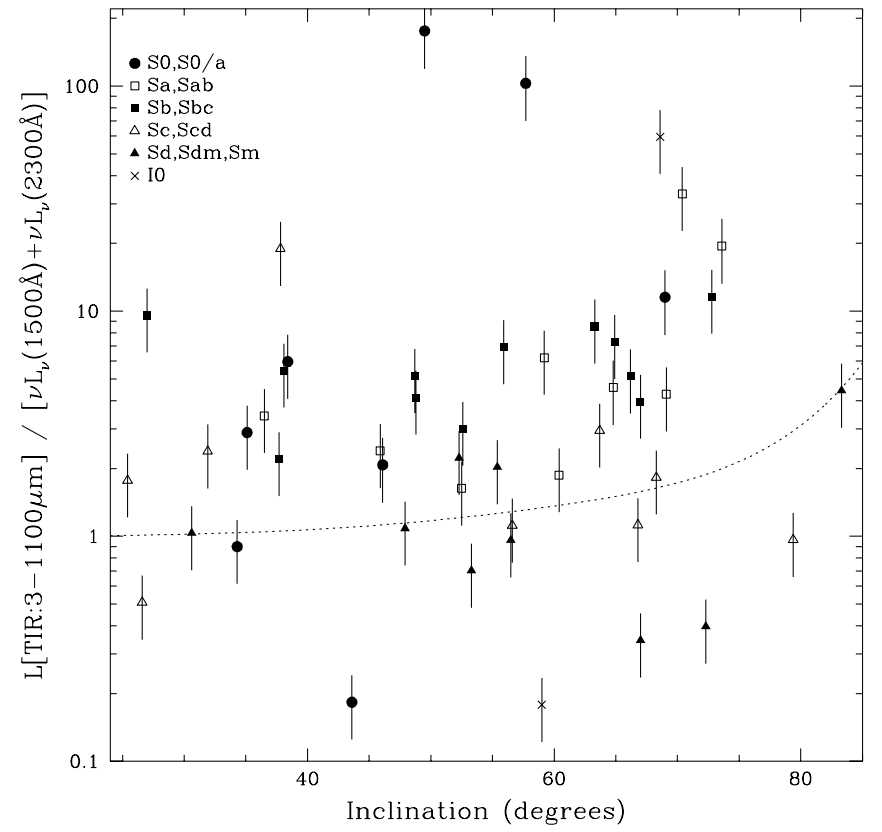

FIG. 4.- Infrared-to-ultraviolet ratio as a function of galaxy disk inclination. The dotted line, normalized to an infrared-to-ultraviolet ratio of unity at zero inclination, shows the expected effect of extinction on the ultraviolet data with changing inclination using the thin-disk model and a central face-on optical depth in the $B$ band of $\tau_{B}^{f}=2$ described in Tuffs et al. (2004). The error bars stem from the observational uncertainties.

galaxies, decreasing their infrared-to-ultraviolet ratios (see, for example, Roussel et al. 2005). Finally, galaxies with smoother $24 \mu \mathrm{m}$ morphologies exhibit cooler far-infrared colors. To see the latter effect more clearly, we show in Figure 9 the ratio of unresolved-to-resolved $24 \mu \mathrm{m}$ emission as a function of far-infrared color. Clearly there is a trend, indicating that the $24 \mu \mathrm{m}$ morphology can, for nearby galaxies, indicate the relative separation be-

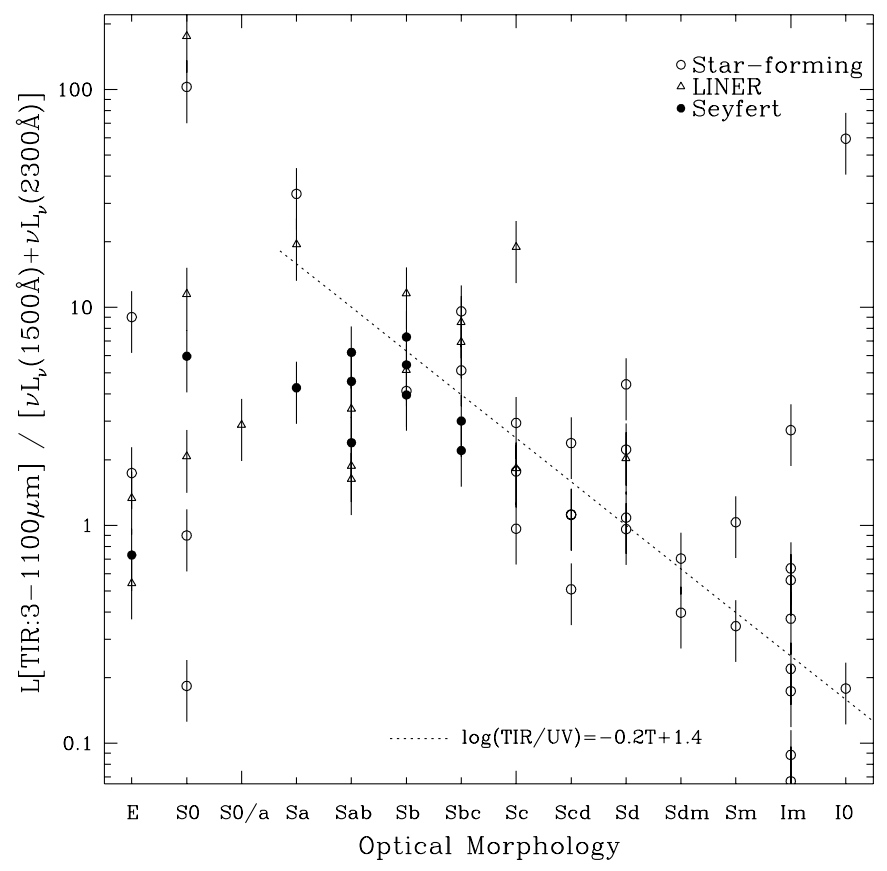

FIG. 5.- Infrared-to-ultraviolet ratio as a function of galaxy optical morphology. The equation provided quantifies the approximate trend with Hubble type for late-type galaxies shown as a dotted line (e.g., $\mathrm{Sa} \rightarrow \mathrm{T}=1, \mathrm{Sb} \rightarrow \mathrm{T}=3$, $\mathrm{Sc} \rightarrow \mathrm{T}=5$, etc.).

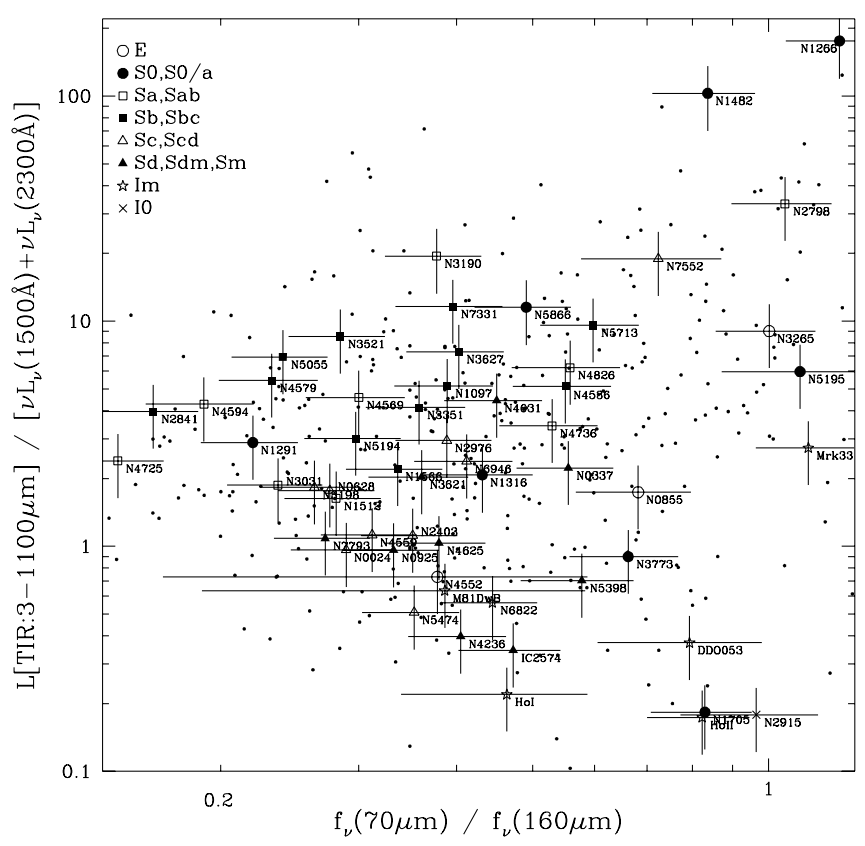

FIG. 6. - Infrared-to-ultraviolet ratio as a function of far-infrared color for the SINGS sample. Data from the GALEX Atlas of Nearby Galaxies (Gil de Paz et al. 2007) are also shown as small data points without error bars. [See the electronic edition of the Journal for a color version of this figure.]

tween interstellar grains and their heating sources. Note that distance contributes but does not dominate as a driver for the effect (the symbol sizes are scaled according to galaxy distance).

\subsection{Specific Star Formation Rate}

One way to parameterize the star formation history of a galaxy is via the star formation rate per stellar mass, or the specific star formation rate (SSFR). Drory et al. (2004) and Feulner et al. (2005), for example, have utilized the specific star formation rate to explore the role of star formation in the growth of stellar mass over cosmic timescales. In this work the specific star formation rate is quantified as a combination of the observed infrared and far-ultraviolet luminosities:

$\operatorname{SSFR}\left(\mathrm{yr}^{-1}\right) \simeq \frac{4.5 \operatorname{TIR}\left(10^{37} \mathrm{~W}\right)+7.1 \nu L_{\nu}(1500 \AA)\left(10^{37} \mathrm{~W}\right)}{0.8 \nu L_{\nu}\left(K_{s}\right)\left(L_{\odot}\right)}$,

based on star formation rate conversion factors from Kennicutt (1998). The numerator in equation (6), applicable for galaxies with continuous star formation occurring on timescales $\gtrsim 10^{8} \mathrm{yr}$, is a more robust way to quantify the star formation rate than relations that are limited to either infrared or ultraviolet luminosities. The infrared luminosity accurately corresponds to the star formation rate only in the limiting case where all of the star formation-related stellar emission is captured by interstellar dust grains. Similarly, the ultraviolet emission can also be a poor measure of the star formation rate, especially when extinction is significant. However, combining both the ultraviolet and infrared luminosities in equation (6) is akin to an extinction-corrected ultraviolet luminosity and thus more effectively recovers the true star formation rate (see also Bell 2003; Iglesias-Páramo et al. 2006). We note that similar values are obtained when using the optimal infrared + ultraviolet star formation rate tracer determined by Hirashita et al. (2003; their eq. [25]), which incorporates parameters that account for the fraction of Lyman continuum luminosity $(f \simeq 0.57$ ), the 


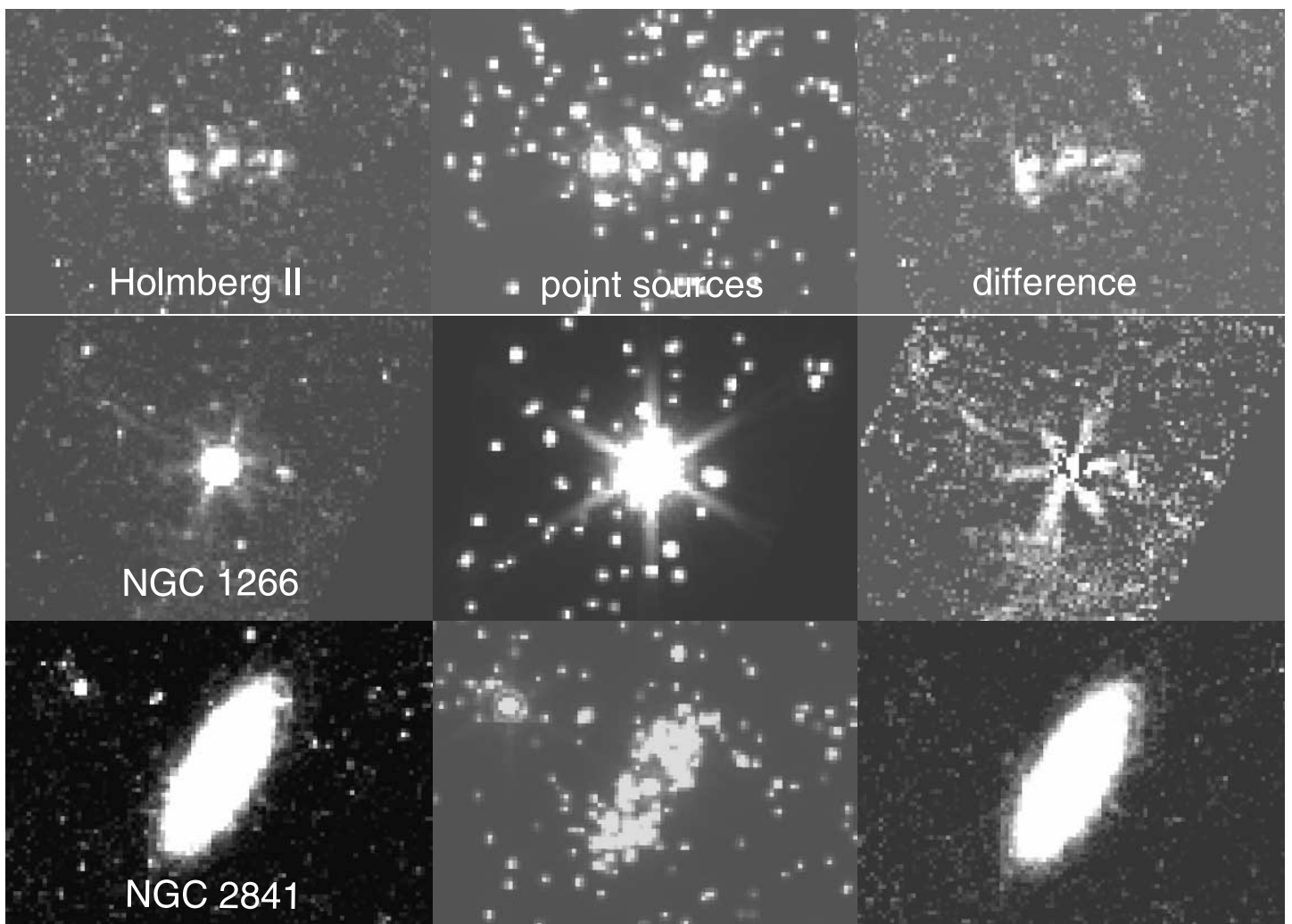

FIG. 7. - Examples of galaxies with clumpy (Holmberg II), unresolved (NGC 1266), and smooth (NGC 2841) $24 \mu \mathrm{m}$ emission. The left, middle, and right panels, respectively, show the original $24 \mu \mathrm{m}$ images, images of the point sources therein, and the differences in the original and point-source images (see $\S 5.3$ ). The images are approximately $700^{\prime \prime}$ across $(\sim 12, \sim 100$, and $\sim 35 \mathrm{kpc}$ for Holmberg II, NGC 1266, and NGC 2841, respectively).

fraction of ultraviolet luminosity absorbed by dust $(\epsilon \simeq 0.53)$, and the fraction of dust heating by stellar populations older than $10^{8} \mathrm{yr}$ $(\eta \simeq 0.40)$. The $K_{s}$ band luminosity in the denominator of equation (6) is equivalent to a stellar mass. Bell et al. (2003), for example, fit stellar population synthesis models to thousands of

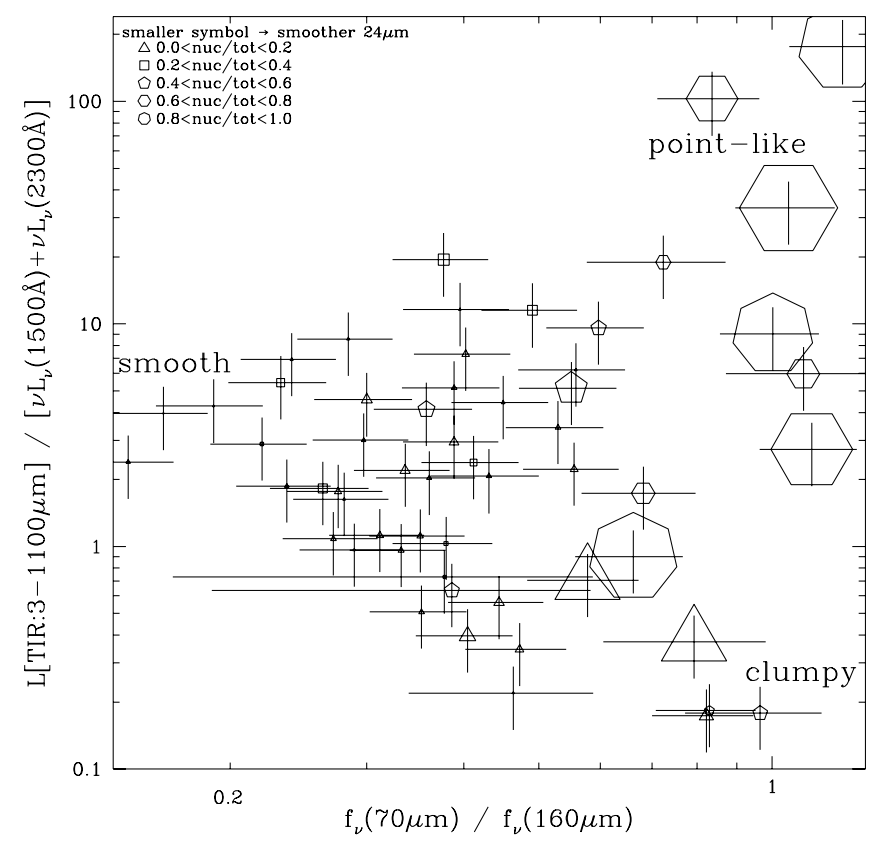

FIG. 8.-Similar to Fig. 6, but with symbol size scaled according to the ratio of unresolved to resolved $24 \mu \mathrm{m}$ emission; the largest symbols have this ratio equal to $\sim 10$. Each data point is also symbolized according to the ratio of nuclear to total $24 \mu \mathrm{m}$ emission (see $\S 5.3$ ).
2MASS plus Sloan Digital Sky Survey optical-near-infrared data sets and find that the distribution of $M_{*} / L_{K_{s}}$ peaks near $\sim 0.8 M_{\odot} L_{\odot}^{-1}$ for a wide range of galactic masses. Gavazzi et al. (1996) also show that the dynamical mass in a galaxy is proportional to the $H$-band luminosity.

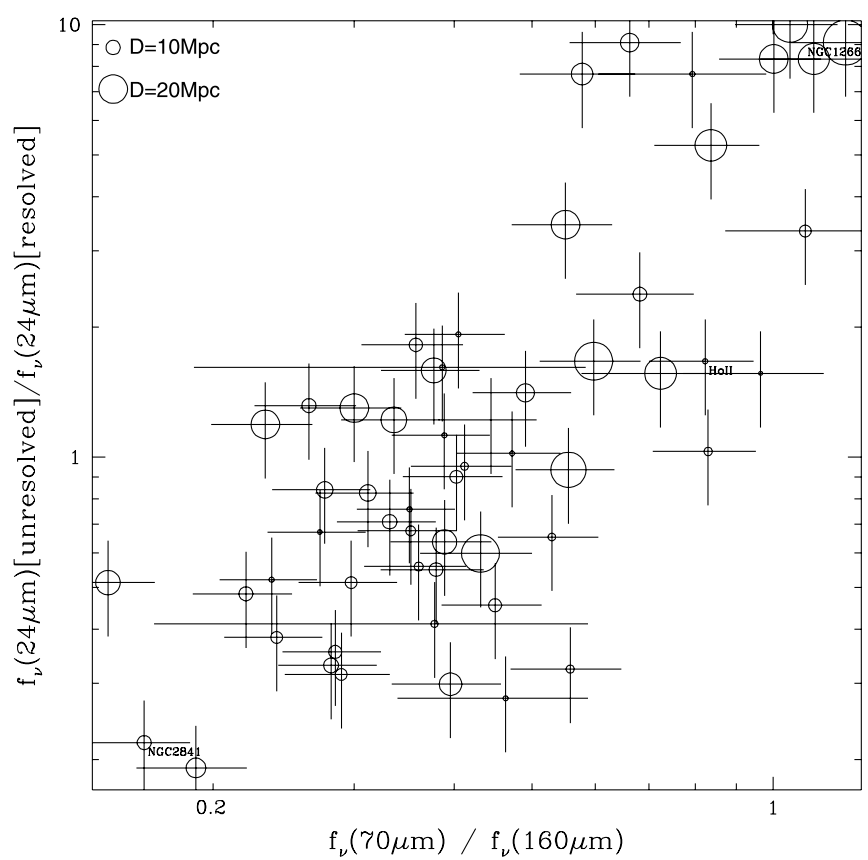

FIG. 9.- Ratio of unresolved to resolved $24 \mu \mathrm{m}$ emission as a function of farinfrared color (see $\S 5.3$ ). A $25 \%$ uncertainty is used for the error bars in the unresolved-to-resolved ratio. The symbol sizes are scaled according to galaxy distance (see legend). 


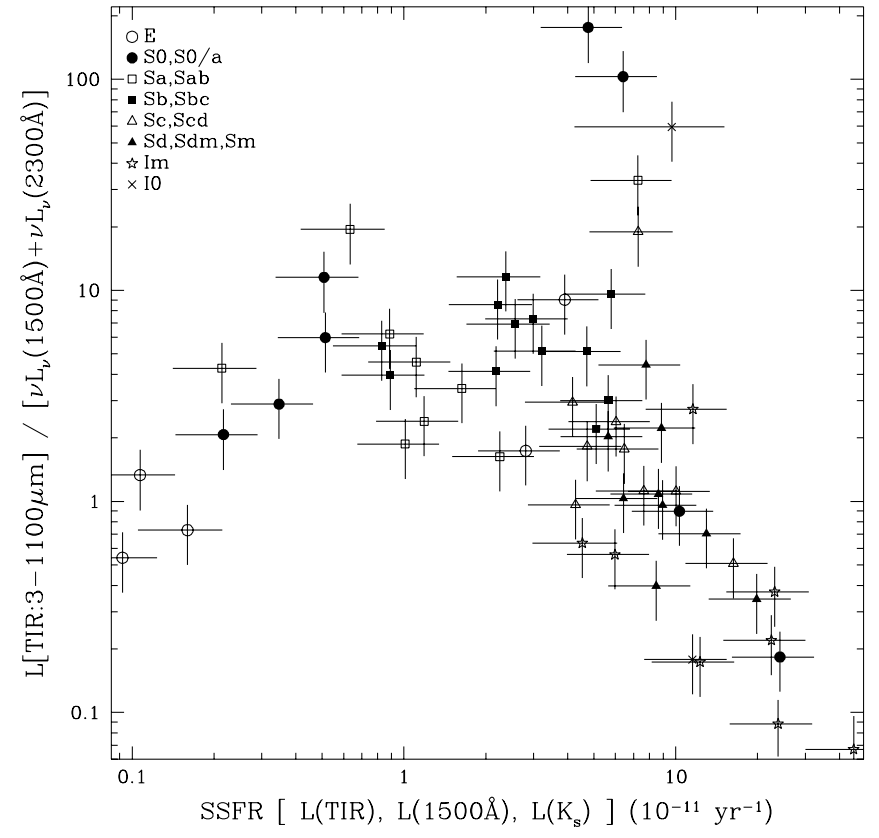

FIG. 10.- Infrared-to-ultraviolet ratio as a function of the specific star formation rate (eq. [6]). The error bars derive from the observational uncertainties plus a $30 \%$ factor assumed for converting the $K_{s}$ luminosity to a stellar mass (see $\S 5.4)$.

Figure 10 presents the interplay between the specific star formation rate, the infrared-to-ultraviolet ratio, and optical morphology. With the exception of a handful of nuclear $24 \mu \mathrm{m}$ sources with high infrared-to-ultraviolet ratios, the SINGS sample shows a general trend in this diagram. Galaxies with low specific star formation rates (SSFR $\lesssim 0.9 \mathrm{yr}^{-1}$ ) are of E, S0, S0/a, or Sa morphologies, consistent with the traditional notion that early-type galaxies exhibit low star formation rates per unit stellar mass (see, e.g., Sandage 1986). These early-type galaxies show increasing infraredto-ultraviolet ratios for increasing specific star formation rates. In contrast, spiral galaxies generally show SSFR $\gtrsim 0.9 \mathrm{yr}^{-1}$, and the later the spiral Hubble type, the larger the specific star formation rate and the smaller the infrared-to-ultraviolet ratio. Note that the numerator in equation (6) overestimates the star formation rate for early-type galaxies, since the bulk of their infrared and ultraviolet luminosities are not due to recently formed stars.

Assuming that equation (6) can be reasonably applied to the SINGS early-type galaxies, in order to observe larger infraredto-ultraviolet ratios (and thus larger dust extinction), increases in the specific star formation must be associated with increased amounts of interstellar dust. On the other hand, increasing the specific star formation rate in late-type galaxies results in smaller infrared-to-ultraviolet ratios: the additional ultraviolet photons in spiral galaxies with high SSFRs tend to more easily escape the galaxies, since their clumpy distribution of dust provides many more sight lines of low optical depth than found in $24 \mu \mathrm{m}$-smooth early-type galaxies. In other words, the increased star formation rate in later type spiral galaxies must lead to a higher density of holes through which ultraviolet photons can escape.

\subsection{Luminosity}

Global parameters related to galaxy structure, star formation history, molecular and atomic gas content, metallicity, etc., are known to trend with $H$-band luminosity, another popular proxy for galaxy stellar mass, especially for late-type galaxies (e.g., Gavazzi et al. 1996; Boselli et al. 2001). Figure 11 displays the

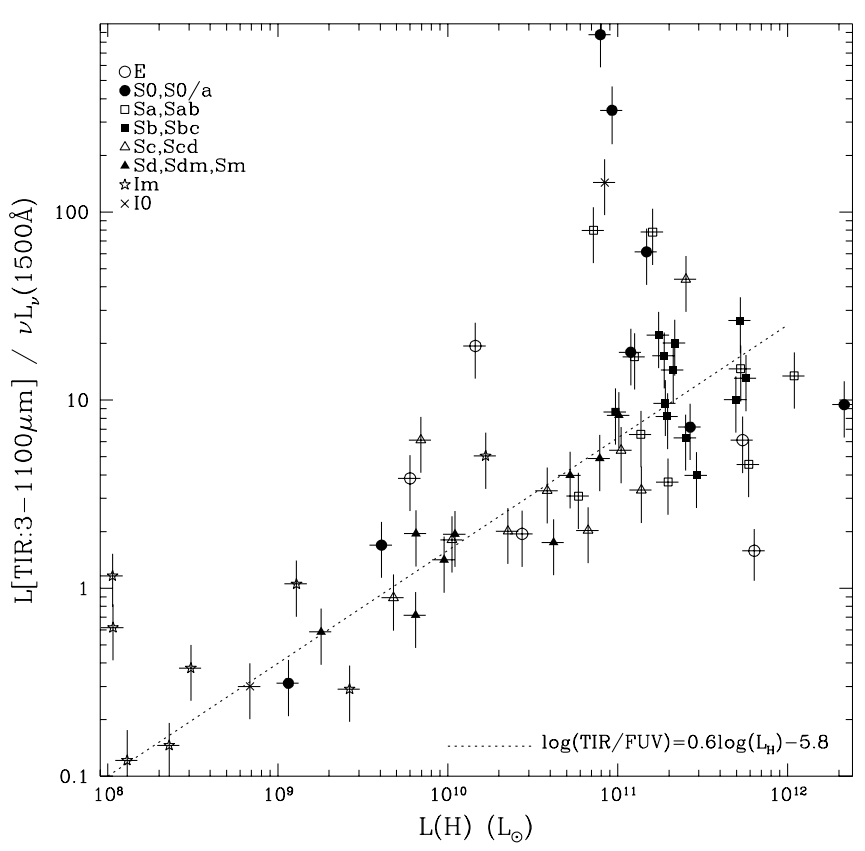

FIG. 11.- Infrared-to-far-ultraviolet ratio as a function of $H$-band luminosity. The dotted line is a fit "by eye" to the general trend.

infrared-to-far-ultraviolet ratio versus 2MASS $H$-band luminosity. A clear correlation is found: more luminous (massive) galaxies show larger infrared-to-far-ultraviolet ratios, consistent with the findings of Cortese et al. (2006). Although most of the low-luminosity dwarfs and early-type galaxies follow the general trend, the data for $\mathrm{E}$ and $\mathrm{S} 0 / \mathrm{S} 0$ a galaxies contribute to increased scatter at high luminosity. This increased scatter for early-type galaxies is not surprising given the large dispersion for these types of galaxies seen in Figure 5 and since the ultravioletemitting stars in the most massive early-type galaxies are generally associated with an old stellar population (see Boselli et al. 2005). Although the older stars might also contribute to the dust heating in massive early-type galaxies, the spectral shapes in Figure 1 suggest that the bulk of the dust heating in these systems is dominated by intermediate-age stars emitting mostly in the visible. In contrast, the spectral shapes for most later type galaxies in Figure 1 indicate that the bulk of the dust heating is being carried out by a younger (bluer) stellar population.

\subsection{Ultraviolet Spectral Slope}

The infrared-to-ultraviolet ratio has been shown to be fairly tightly correlated with the ultraviolet spectral slope in starburst galaxies, an important discovery that allows the extinction at ultraviolet wavelengths to be estimated from ultraviolet spectral data alone (e.g., Calzetti et al. 1994; Calzetti 1997; Meurer et al. 1999). A starburst galaxy is defined here as a galaxy experiencing prodigious, recent star formation (perhaps triggered by an encounter) at a rate that cannot be sustained over the lifetime of the galaxy. Nonstarbursting galaxies have also been studied in the context of infrared-to-ultraviolet ratio and ultraviolet spectral slope, but their data show a larger dispersion, with normal starforming and quiescent systems exhibiting redder ultraviolet spectra and/or lower infrared-to-ultraviolet ratios (e.g., Buat et al. 2002, 2005; Bell 2002; Kong et al. 2004; Gordon et al. 2004; Burgarella et al. 2005; Calzetti et al. 2005; Seibert et al. 2005; Cortese et al. 2006; Boissier et al. 2007; Gil de Paz et al. 2007). The intrinsic ultraviolet spectral slope is quite sensitive to the effective age of the stellar population, leading Calzetti et al. (2005) 


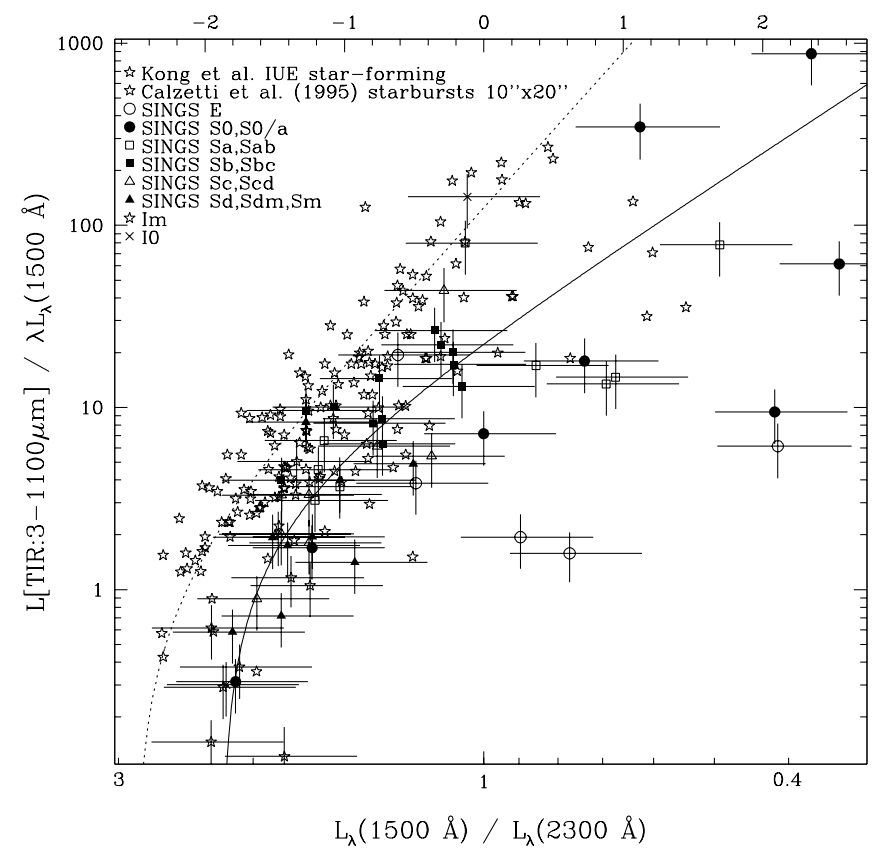

FIG. 12.- Infrared-to-far-ultraviolet ratio as a function of ultraviolet spectral slope. Normal star-forming and starbursting galaxies from Kong et al. (2004) and Calzetti et al. (1995) are plotted in addition to the SINGS data points. The dotted line is that for starbursting galaxies from Kong et al. (2004), and the solid line is applicable to normal star-forming galaxies (Cortese et al. 2006). [See the electronic edition of the Journal for a color version of this figure.]

to suggest that the evolved, nonionizing stellar population $(\sim 50-$ $100 \mathrm{Myr}$ ) dominates the ultraviolet emission in normal systems, in contrast to current star formation processes dominating the ultraviolet emission in starbursts. The increased diversity in the ultraviolet spectral slopes for evolved stellar populations manifests itself as a larger dispersion for quiescent and normal star-forming galaxies in plots of the infrared-to-ultraviolet ratio as a function of ultraviolet spectral slope. Interestingly, Boissier et al. (2007) use azimuthally averaged radial profiles, and after excluding emission from the bulge/nucleus, they find that the relation between infrared-to-ultraviolet and ultraviolet slope tightens up compared with the one obtained using the integrated data. This result is consistent with the interpretation of Calzetti et al. (2005) either if the evolved stellar populations in normal star-forming galaxy bulges cause the increased scatter compared to the starburst trend or if azimuthally averaging smooths over small-scale effects such as the heating of dust in one region by ultraviolet light from a different nearby region.

Figure 12 displays a diagram of spatially integrated infraredto-ultraviolet ratios and ultraviolet spectral slopes. Normal starforming and starbursting galaxies from Kong et al. (2004) and Calzetti et al. (1995) are plotted in addition to the SINGS data points. The dotted line is that for starbursting galaxies from Kong et al. (2004), and the solid line is applicable to normal star-forming galaxies of type $\mathrm{Sa}$ or later (Cortese et al. 2006). Similar to what has been found for other samples of nonstarbursting galaxies, the SINGS data set shows more scatter in this diagram and the galaxies are redder in their ultraviolet spectral slope compared to starburst galaxies. Inspection of the distribution as a function of SINGS optical morphology, however, shows that the 14 reddest SINGS galaxies are type Sab or earlier, a result that is perhaps expected for systems with quenched star formation rate histories; the early-type galaxies in SINGS contribute to most of the observed scatter, such that the trend for the subset of just late-type SINGS galaxies shows a dispersion comparable to that for star- bursts. The 14 reddest SINGS galaxies also significantly differ from even the normal galaxy curve, but this is consistent with the fact that Cortese et al. (2006) excluded types earlier than Sa for their analysis. Finally, although the SINGS sample shows very large dispersion and does not as a whole match the starburst trend, we have verified that the starburst subset of SINGS (e.g., Mrk 33, NGC 1705, NGC 2798, NGC 3034, NGC 7552) does indeed match the canonical starburst trend.

\section{DISCUSSION AND SUMMARY}

The ultraviolet-to-radio broadband SEDs are presented for the 75 galaxies in the Spitzer Infrared Nearby Galaxies Survey, a collection of galaxies that broadly samples the wide variety of galaxy morphologies, luminosities, colors, and metallicities seen in the local universe. The infrared-to-ultraviolet ratio is explored in conjunction with several global parameters. An interesting empirical finding is that systems with cooler dust show a restricted range of infrared-to-ultraviolet ratios $(\sim 0.5 \mathrm{dex})$, while systems with warm global far-infrared colors exhibit a large range of infrared-to-ultraviolet ratios $(\sim 3 \mathrm{dex})$. To put it another way, the cold dust systems in the SINGS sample show average ultraviolet extinctions; no cold galaxy is particularly optically thick or thin. There remains the possibility that part of this distribution is attributable to selection effects, but we use the morphology from MIPS $24 \mu \mathrm{m}$ imaging to interpret this distribution to result from the relative distribution of dust grains and their heating sources. Nearby galaxies with globally cooler dust appear smoother at $24 \mu \mathrm{m}$, from which we infer that the dust grains are well mixed throughout the interstellar medium and not concentrated near sites of active star formation. On the other hand, galaxies with elevated $f_{\nu}(70 \mu \mathrm{m}) / f_{\nu}(160 \mu \mathrm{m})$ ratios appear as one or a handful of clumps at $24 \mu \mathrm{m}$ and thus have much of their dust considerably closer to heating sources. The observed range in infrared-to-ultraviolet ratio is also related to the $24 \mu \mathrm{m}$ morphology, from which the density of available clean lines of sight for ultraviolet photons to escape can be inferred. The dust distribution in galaxies appearing as a single clump at $24 \mu \mathrm{m}$ heavily enshrouds the heating sources (high infrared-to-ultraviolet ratios), galaxies with multiple clumps at $24 \mu \mathrm{m}$ provide a large number of low optical depth lines of sight along which ultraviolet photons can escape (low infrared-to-ultraviolet ratios), and a smooth distribution at $24 \mu \mathrm{m}$ implies a dust distribution that provides an intermediate number of low optical depth lines of sight (average infrared-to-ultraviolet ratios). Detailed studies of the relative distributions of the infrared emission and the ionizing radiation fields in SINGS galaxies have been carried out in IC 2574 (Cannon et al. 2005), NGC 1705 (Cannon et al. 2006a), and NGC 6822 (Cannon et al. 2006b). These dwarf galaxies appear as multiple clumps at $24 \mu \mathrm{m}$ and show low optical extinctions and highly variable ratios of $\mathrm{H} \alpha$ to infrared (i.e., significant ultraviolet photon leakage), consistent with our expectation that multiclump $24 \mu \mathrm{m}$ galaxies should have warm far-infrared colors and low global infrared-to-ultraviolet ratios.

A principal component analysis of the SINGS broadband spectra indicates that most of the sample's large broadband spectral variations stem from two underlying components, one typical of a galaxy with a low infrared-to-ultraviolet ratio $(88 \%$ of the sample variation) and one indicative of a galaxy with a high infrared-toultraviolet ratio ( $7 \%$ of the sample variation). The implication is that the star formation history (i.e., the specific star formation rate, the birthrate parameter, or some other measure of the current to past star formation rate) may be the dominant regulator of the broadband spectral variations between galaxies. From a morphological standpoint, we find that much of the dispersion in plots 
such as infrared-to-ultraviolet versus ultraviolet spectral slope (Fig. 12) stems from early-type galaxies, which have significantly redder ultraviolet spectra than other galaxy types. In fact, the galaxies with the highest optical-to-infrared ratios, the smallest specific star formation rates, and the reddest ultraviolet slopes are all early-type galaxies (see Figs. 1, 10, and 12, respectively).

Evidence for the star formation history regulating spectral variations is found in a striking trend in the infrared-to-ultraviolet ratio as a function of the specific star formation rate (Fig. 10). Early-type galaxies show higher ratios of infrared to ultraviolet (higher dust extinction) for larger specific star formation rates, implying that the specific star formation rate in elliptical and S0 galaxies is closely tied to the amount of dust. Conversely, spiral galaxies show lower infrared-to-ultraviolet ratios (lower dust extinction) for higher specific star formation rates, suggesting that the specific star formation rate in elliptical galaxies is linked to the distribution of dust: in spiral galaxies a larger number of holes are created for increased star formation activity, holes through which ultraviolet light more easily passes out of galaxies.

In a study of 99,088 galaxies from the Sloan Digital Sky Survey, Obric et al. (2006) find that the GALEX, Sloan, and 2MASS data "form a nearly one parameter family." In particular, they can predict with $20 \%$ accuracy the 2 MASS $K_{s}$ flux using just the Sloan $u$ and $r$ fluxes. In addition, they can predict to within a factor of 2 certainty the IRAS $60 \mu \mathrm{m}$ flux based on the Sloan broadband data. Such simple optical-infrared correlations are not seen for SINGS galaxies. However, Obrić et al. (2006) are only able to identify IRAS fluxes for less than $2 \%$ of their sample, and this subset is strongly biased to optically blue galaxies. The SINGS sample, although far smaller in size, provides complete panchromatic information for a far more diverse ensemble of galaxies and is thus much less biased to a particular subset of the local galaxy population.

Support for this work, part of the Spitzer Space Telescope Legacy Science Program, was provided by NASA through contract 1224769 issued by the Jet Propulsion Laboratory, California Institute of Technology under NASA contract 1407. A. G. d. P. is financed by the MAGPOP EU Marie Curie Research Training Network and the Spanish Programa Nacional de Astronomía y Astrofísica under grant AYA2003-01676. We are thankful for the hard work put in by the instrument teams and the Spitzer Science Center. We gratefully acknowledge NASA's support for construction, operation, and science analysis for the GALEX mission, developed in cooperation with the Centre National d'Etudes Spatiales of France and the Korean Ministry of Science and Technology. This research has made use of the NASA/IPAC Extragalactic Database, which is operated by JPL/Caltech, under contract with NASA. This publication makes use of data products from the Two Micron All Sky Survey, which is a joint project of the University of Massachusetts and IPAC/Caltech, funded by NASA and the National Science Foundation.
Adelberger, K. L., \& Steidel, C. C. 2000, ApJ, 544, 218

Bauer, F. E., Condon, J. J., Thuan, T. X., \& Broderick, J. J. 2000, ApJS, 129, 547

Bell, E. F. 2002, ApJ, 577, 150 2003, ApJ, 586, 794

Bell, E. F., Gordon, K. D., Kennicutt, R. C., \& Zaritsky, D. 2002, ApJ, 565, 994 Bell, E. F., McIntosh, D. H, Katz, N., \& Weinberg, M. D. 2003, ApJS, 149, 289 Boissier, S., et al. 2007, ApJ, in press

Boselli, A., \& Gavazzi, G. 1994, A\&A, 283, 12

Boselli, A., Gavazzi, G., Donas, J., \& Scodeggio, M. 2001, AJ, 121, 753

Boselli, A., Gavazzi, G., \& Sanvito, G. 2003, A\&A, 402, 37

Boselli, A., et al. 2005, ApJ, 629, L29

Bruzual, A. G., Magris, G., \& Calvet, N. 1988, ApJ, 333, 673

Buat, V., Boselli, A., Gavazzi, G., \& Bonfanti, C. 2002, A\&A, 383, 801

Buat, V., \& Xu, C. 1996, A\&A, 306, 61

Buat, V., et al. 2005, ApJ, 619, L51

Burgarella, D., Buat, V., \& Iglesias-Páramo, J. 2005, MNRAS, 360, 1413

Calzetti, D. 1997, AJ, 113, 162

Calzetti, D., Bohlin, R. C., Kinney, A. L., Storchi-Bergmann, T., \& Heckman,

T. M. 1995, ApJ, 443, 136

Calzetti, D., Kinney, A. L., \& Storchi-Bergmann, T. 1994, ApJ, 429, 582

Calzetti, D., et al. 2005, ApJ, 633, 871

Cannon, J. M., et al. 2005, ApJ, 630, L37

2006a, ApJ, 647, 293

2006b, ApJ, 652, 1170

Chapman, S. C., Smail, I., Ivison, R. J., Helou, G., Dale, D. A., \& Lagache, G. 2002, ApJ, 573, 66

Condon, J. J. 1987, ApJS, 65, 485

Condon, J. J., Cotton, W. D., Greisen, E. W., Yin, Q. F., Perley, R. A., Taylor, G. B., \& Broderick, J. J. 1998, AJ, 115, 1693

Condon, J. J., Helou, G., Sanders, D. B., \& Soifer, B. T. 1990, ApJS, 73, 359 Cortese, L., et al. 2006, ApJ, 637, 242

Dale, D. A., Giovanelli, R., Haynes, M. P., Scodeggio, M., Hardy, E., \& Campusano, L. E. 1997, AJ, 114, 455

Dale, D. A., \& Helou, G. 2002, ApJ, 576, 159

Dale, D. A., Helou, G., Contursi, A., Silbermann, N. A., \& Kolhatkar, S. 2001, ApJ, 549, 215

Dale, D. A., et al. 2005, ApJ, 633, 857

Deeming, T. F. 1964, MNRAS, 127, 493

de Vaucouleurs, G., de Vaucouleurs, A., Corwin, H. G., Buta, R. J., Paturel, G., \& Fouqué, P. 1991, Third Reference Catalogue of Bright Galaxies (New York: Springer)

\section{REFERENCES}

Diolaiti, E., Bendinelli, O., Bonaccini, D., Close, L., Currie, D., \& Parmeggiani, G. 2000, A\&AS, 147, 335

Draine, B. T. 2003, ARA\&A, 41, 241

Drory, N., Bender, R., Feulner, G., Hopp, U., Maraston, C., Snigula, J., \& Hill, G. J. 2004, ApJ, 608, 742

Engelbracht, C. W., Gordon, K. D., Rieke, G. H., Werner, M. W., Dale, D. A., \& Latter, W. B. 2005, ApJ, 628, L29

Feulner, G., Goranova, Y., Drory, N., Hopp, U., \& Bender, R. 2005, MNRAS, 358, L1

Fukugita, M., Nakamura, O., Turner, E. L., Helmboldt, J., \& Nichol, R. C. 2004, ApJ, 601, L127

Galliano, F., Madden, S. C., Jones, A. P., Wilson, C. D., \& Bernard, J. P. 2005 , A\&A, 434, 867

Gavazzi, G., Pierini, D., \& Boselli, A. 1996, A\&A, 312, 397

Gil de Paz, A., et al. 2007, ApJ, in press

Giovanelli, R., Haynes, M. P., Salzer, J. J., Wegner, G., da Costa, L. N., \& Freudling, W. 1995, AJ, 110, 1059

Gordon, K. D., Clayton, G. C., Witt, A. N., \& Misselt, K. A. 2000, ApJ, 533, 236

Gordon, K. D., et al. 2004, ApJS, 154, 215

Helou, G., et al. 2004, ApJS, 154, 253

Hinz, et al. 2004, ApJS, 154, 259

Hirashita, H., Buat, V., \& Inoue, A. K. 2003, A\&A, 410, 83

Hummel, E. 1980, A\&AS, 41, 151

Hunter, D. A., \& Gallagher, J. S., III 1986, PASP, 98, 5

Iglesias-Páramo, J., et al. 2006, ApJS, 164, 38

Inoue, A. K., Buat, V., Burgarella, D., Panuzzo, P., Takeuchi, T. T., \& IglesiasPáramo, J. 2006, MNRAS, 370, 380

Jarret, T. H., Chester, T., Cutri, R., Schneider, S. E., \& Huchra, J. P. 2003, AJ, 125,525

Kennicutt, R. C. 1998, ARA\&A, 36, 189

Kennicutt, R. C., et al. 2003, PASP, 115, 928

Kong, X., Charlot, S., Brinchmann, J., \& Fall, S. M. 2004, MNRAS, 349, 769

Krist, J. 2002, Tiny Tim/SIRTF User's Guide (Pasadena: Spitzer Science Center)

Kroupa, P. 2002, Science, 295, 82

Kuchinski, L. E., Terndrup, D. M., Gordon, K. D., \& Witt, A. N. 1998, AJ, 115, 1438

Li, A., \& Draine, B. T. 2001, ApJ, 554, 778

Martin, D. C., et al. 2005, ApJ, 619, L1

Meurer, G. R., Heckman, T. M., \& Calzetti, D. 1999, ApJ, 521, 64 
Murphy, E. J., et al. 2006, ApJ, 638, 157

Obrić, M., et al. 2006, MNRAS, 370, 1677

O’Connell, R. W. 1999, ARA\&A, 37, 603

Panagia, N. 1973, AJ, 78, 929

Rich, R. M., et al. 2005, ApJ, 619, L107

Roussel, H., Gil de Paz, A., Seibert, M., Helou, G., Helou, G., Madore, B. F., \& Martin, C. 2005, ApJ, 632, 227

Roussel, H., Helou, G., Beck, R., Condon, J. J., Bosma, A., Matthews, K., \& Jarrett, T. H. 2003, ApJ, 593, 733

Rowan-Robinson, M., et al. 2005, AJ, 129, 1183

Sajina, A., Scott, D., Dennefeld, M., Dole, H., Lacy, M., \& Lagache, G. 2006, MNRAS, 369, 939

Sandage, A. 1986, A\&A, 161, 89

Schlegel, D. J., Finkbeiner, D. P., \& Davis, M. 1998, ApJ, 500, 525
Schmitt, H. R., Calzetti, D., Armus, L., Giavalisco, M., Heckman, T. M., Kennicutt, R. C., Leitherer, C., \& Meurer, G. R. 2006, ApJ, 643, 173

Seibert, M., et al. 2005, ApJ, 619, L55

Tuffs, R. J., Popescu, C. C., Völk, H. J., Kylafis, N. D., \& Dopita, M. A. 2004, A\&A, 419, 821

van Zee, L., Haynes, M. P., \& Salzer, J. J. 1997, AJ, 114, 2479

Vazquez, G. A., \& Leitherer, C. 2005, ApJ, 621, 695

Walter, F., et al. 2006, ApJ, submitted

Wright, A., \& Otrupcek, R. 1990, Parkes Catalogue (Australian Telescope National Facility)

Xilouris, E. M., Madden, S. C., Galliano, F., Vigroux, L., \& Sauvage, M. 2004, A\&A, 416, 41

Yi, S. K., et al. 2005, ApJ, 619, L111

Yun, M. S., Reddy, N. A., \& Condon, J. J. 2001, ApJ, 554, 803 